\title{
RDUS
}

Revue de DROIT

UNIVERSITÉ DE SHERBROOKE

Titre : NON-INDÉPENDANCE ET AUTONOMIE DE LA NORME D'ÉGALITÉ QUÉBÉCOISE : DES CONCEPTS ॥ FONDATEURS ॥ QUI MÉRITENT D'ÊTRE MIEUX CONNUS

Auteur(s) : $\quad$ David ROBITAILLE

Revue : $\quad$ RDUS, 2004-2005, volume 35, numéro 1

Pages: $\quad 103-143$

ISSN : $\quad 0317-9656$

Éditeur : $\quad$ Université de Sherbrooke. Faculté de droit.

URI : $\quad$ http://hdl.handle.net/11143/11934

DOI : $\quad$ https://doi.org/10.17118/11143/11934 
Page vide laissée intentionnellement. 


\title{
ARTICLE
}

\section{NON-INDÉPENDANCE ET AUTONOMIE DE LA NORME D’ÉGALITÉ QUÉBÉCOISE : DES CONCEPTS «FONDATEURS» QUI MÉRITENT D'ÊTRE MIEUX CONNUS*}

\author{
par David ROBITAILLE**
}

L'article 10 de la Charte des droits et libertés de la personne en est à un tournant majeur de sa courte histoire. Les années 1980 ont d'abord été celles de la doctrine, plusieurs auteurs contribuant à cette époque à dégager le sens et la portée de la norme d'égalité qu'il énonce. L'un des constats qui se dégageait alors consistait en la reconnaissance du champ normatif du droit à la nondiscrimination dont l'application est limitée aux droits et libertés de la personne. Le plus haut tribunal de la province prenait acte de cet aspect de l'article 10 lorsqu'il affirmait en 1994 qu'il constituait une «modalité de particularisation» d'autres droits ou libertés. Si tous ont reconnu de façon générale ce trait caractéristique, aucun auteur, à notre connaissance, n'a présenté d'étude approfondie sur ses conséquences véritables. Il s'agit pourtant d'un aspect de l'article 10 qui en définit fondamentalement le rôle dans la Charte québécoise. Ce texte sera ainsi l'occasion de mettre en exergue la relation particulière que vit la norme d'égalité avec les autres droits et libertés consacrés dans la Charte. Nous poserons inévitablement un regard sur la nouvelle interprétation que fait la Cour d'appel de l'article 10 depuis l'arrêt Law c. Canada rendu par la Cour suprême sur l'article 15 de la Charte canadienne des droits et libertés. Nous constaterons ainsi l'impasse dans laquelle se trouve maintenant la norme d'égalité québécoise.

* $\quad$ Ce texte est issu du mémoire de maîtrise que le soussigné a déposé en décembre 2002 à la Faculté de droit de l'Université Laval. Ce dernier tient à remercier son directeur de recherche, monsieur Ghislain Otis, professeur titulaire à la Faculté de droit de l'Université Laval, pour sa disponibilité, sa générosité et sa bonne humeur constantes, ainsi que pour ses suggestions en vue de la présente publication. Plusieurs idées exprimées dans ce texte, dont certaines proviennent directement du professeur Otis, sont par ailleurs le fruit des discussions entre celui-ci et l'auteur dans le cadre de son cheminement à la maîtrise. L'auteur est également très reconnaissant envers la Faculté de droit de l'Université Laval qui, par l'entremise du Fonds d'enseignement et de recherche, lui a octroyé une importante bourse d'étude.

**. LL.L. (Ottawa), LL.M. (Laval), doctorant à la Faculté de droit de l'Université d'Ottawa. 
Section 10 of the Quebec Charter of Human Rights and Freedoms has reached a turning point in its short history. The 80's were characterized by doctrinal writings in which several authors contributed to fleshing out the meaning and scope of the equality clause. One of the positions advanced and subsequently confirmed in 1994 by the highest court of the province, sets out the ambit of the right to non-discrimination, the application of which is limited to the rights and freedoms of the person. In other words, section 10 is a «form of particularization» of other rights and freedoms. Although this feature has been generally accepted, no writer, as far as we know, has made a thorough study of its actual consequences. This aspect is nevertheless essential since it defines the very role of section 10 in the Charter. The purpose of this paper is to present the particular relationship between the equality standard and the other rights and freedoms recognized by the Charter. One must inevitably take into consideration the most recent interpretation of section 10 by the Quebec Court of Appeal in light of the Supreme Court decision in Law v. Canada dealing with section 15 of the Canadian Charter of Rights and Freedoms. The Quebec equality clause would appear to be at an impasse 
Non-indépendance et autonomie de la norme

(2004) 35 R.D.U.S. d'égalité québécoise : des concepts «fondateurs» qui méritent d'être mieux connus

\section{SOMMAIRE}

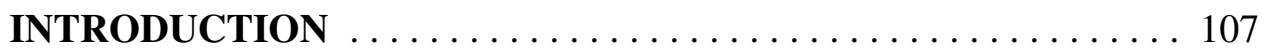

1. La non-indépendance de l'article 10 de la Charte : aux

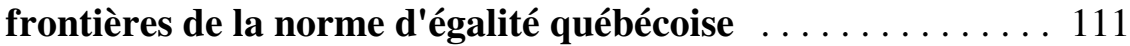

A. L'application large et libérale de la norme d'égalité pour pallier sa limitation inhérente de non-indépendance ... 119

B. La quasi-indépendance de la norme d'égalité par l'utilisation du concept de dignité en matière de discrimination . . . . . . . . . . . . . . . . 124

\section{L'indispensable autonomie de la norme d'égalité québécoise}

. . . . . . . . . . . . . . . . . . . . . . . . . . . . . . . 128

A. L'importance capitale de la norme autonome d'égalité lorsque rattachée à un droit économique ou social . . . . . 131

B. L'autonomie de la norme d'égalité menacée par l'utilisation du concept de dignité en matière de discrimination et encore mal comprise par certains .... 136 

Non-indépendance et autonomie de la norme
(2004) 35 R.D.U.S. $\quad$ d'égalité québécoise : des concepts «fondateurs» qui méritent d'être mieux connus

\section{INTRODUCTION}

En vigueur depuis 1975, la Charte des droits et libertés de la personne ${ }^{1}$ consacre en son article 10 le droit à la non-discrimination de la façon suivante :

Toute personne a droit à la reconnaissance et à l'exercice, en pleine égalité, des droits et libertés de la personne, sans distinction, exclusion ou préférence fondée sur la race, la couleur, le sexe, la grossesse, l'orientation sexuelle, l'état civil, l'âge sauf dans la mesure prévue par la loi, la religion, les convictions politiques, la langue, l'origine ethnique ou nationale, la condition sociale, le handicap ou l'utilisation d'un moyen pour pallier ce handicap.

Il y a discrimination lorsqu'une telle distinction, exclusion ou préférence a pour effet de détruire ou de compromettre ce droit ${ }^{2}$.

Au début des années 1980 et vers la fin de la même décennie, cette disposition a fait l'objet de plusieurs études qui visaient à en dégager le sens et la portée ${ }^{3}$. Un auteur avançait alors que l'article 10 constituait une «modalité de particularisation $»^{4}$ des droits et libertés de la personne. En 1994, la Cour d'appel du Québec confirmait en ces termes cette position dans l'arrêt de principe Commission scolaire St-Jean-sur-Richelieu c. Commission des droits de la personne du Québec:

Lorsque l'égalité n'est qu'une modalité de particularisation d'un autre droit, il n'est pas nécessaire que sa violation constitue une négation de ce droit pour donner ouverture à un remède. Il suffit que soit établie,

1. L.R.Q. c. C-12 (ci-après Charte).

2. Ibid., art. 10.

3. Voir notamment : P. Blache, «Les méandres du concept d'égalité et la Charte québécoise des droits et libertés de la personne» (1986) 17 R.D.U.S. 85; P. Carignan, «L'égalité dans le droit : une méthode d'approche appliquée à l'article 10 de la Charte des droits et libertés de la personne» (1987) 21 R.J.T. 491; M. Caron, «Les concepts d'égalité et de discrimination dans la Charte des droits et libertés de la personne», dans Service de la formation permanente, Barreau du Québec, Développements récents en droit administratif (1993), Cowansville (Qc), Yvon Blais à la p. 39; D. Proulx, «Égalité et discrimination dans la Charte des droits et libertés de la personne : étude comparative» (1980) 10 R.D.U.S. 381.

4. $\quad$ P. Carignan, ibid. à la p. 526. 
dans la détermination des modalités de ce droit ou de cette liberté, une distinction incompatible avec la règle de l'égalité 5 .

En effet, comme la majorité des auteurs l'ont reconnu, et tel que l'indique son libellé, l'article 10 ne consacre pas un droit général à l'égalité mais plutôt le droit à l'égale reconnaissance ou exercice des autres droits et libertés de la personne reconnus dans la Charte ${ }^{6}$. Autrement dit, la norme d'égalité québécoise se trouve à faire partie intégrante ${ }^{7}$ des droits et libertés de la

5. Commission scolaire St-Jean-sur-Richelieu c. Commission des droits de la personne du Québec, [1994] R.J.Q. 1227 à la p. 1243 (C.A.) (ci-après Commission scolaire St-Jean-surRichelieu). Sur ce point, voir aussi : Habachi c. Commission des droits de la personne, [1999] R.J.Q. 2522 aux pp. 2537 et 2538 (C.A.) (ci-après Habachi); Commission des droits de la personne et des droits de la jeunesse c. Poulin, T.D.P.Q., Québec, 200-53-000016-001, 2001-03-14, AZ-50085390 à la p. 8 du texte intégral (ci-après Poulin); Commission des droits de la personne et des droits de la jeunesse c. Bertrand, [2001] R.J.Q. 1684 aux pp. 1690 et 1691 (T.D.P.Q.) (ci-après Bertrand); Commission des droits de la personne et des droits de la jeunesse et Bouffard, T.D.P.Q., Saint-François, 450-53-000001-988, 1999-0430, AZ-50065697 au para. 11 (ci-après Bouffard); H. Brun et G. Tremblay, Droit constitutionnel, 4 éd., Cowansville (Qc), Yvon Blais, 2002 à la p. 1073; P. Carignan, supra note 3 aux pp. 527 et 530; M. Caron, supra note 3 à la p. 52; D. Proulx, supra note 3 à la p. 497 et $\mathrm{s}$.

6. Gosselin c. Québec (Procureur général), [2002] C.S.C. 84 au para. 430 (J. Lebel); Commission scolaire St-Jean-sur-Richelieu, ibid. à la p. 1243; Bertrand, ibid. à la p. 1690; Québec (Ville) c. Commission des droits de la personne du Québec, [1989] R.J.Q. 831 à la p. 844 (C.A.); Rondeau c. Syndicat des employé(e)s du Centre de services sociaux du Montréal Métropolitain, T.D.P.Q., Montréal, 500-53-000019-941, 1995-03-29, J. Brossard; P. Blache, «Étude comparative de l'évolution des normes antidiscriminatoires ou égalitaires des articles 15 de la Charte canadienne des droits et libertés et 10 de la Charte des droits et libertés de la personne : convergences et divergences» ( $\mathrm{n}^{\circ}$ spécial mars 2003) R. du B. 151 à la p. 166; H. Brun et G. Tremblay, ibid. à la p. 1106; M. Caron, supra, note 3 aux pp. 42 à 44; G. Otis et C. Brunelle, «La Charte des droits et libertés de la personne et la tenue vestimentaire à l'école publique» (1995) 36 C. de D. 599; D. Proulx, supra note 3 à la p. 480 et s.; D. Proulx, «Le concept de dignité et son usage en contexte de discrimination: deux Chartes, deux modèles» ( ${ }^{\circ}$ spécial mars 2003) R. du B. 485 aux pp. 516 à 518. Le champ d'application de l'article 10 est tout de même très vaste en raison de l'ampleur des droits et libertés garantis : C. Brunelle, «Les droits et libertés dans le contexte civil» dans Collection de droit 2001-02, École du Barreau du Québec, vol. 7, Droit public et administratif, Cowansville (Qc), Yvon Blais, 2001 à la p. 53. L'article 50 de la Charte permettrait au surplus à l'article 10 de s'appliquer à des droits et libertés non reconnus par celle-ci : H. Brun et G. Tremblay, ibid. aux pp. 1106-1107; P. Carignan, supra note 3 à la p. 528; D. Proulx, supra note 3 aux pp. 486 à 488 .

7. P. Carignan, supra note 3 à la p. 528. 
Non-indépendance et autonomie de la norme
(2004) 35 R.D.U.S. $\quad$ d'égalité québécoise : des concepts «fondateurs» qui méritent d'être mieux connus

personne et renforce ou complète leur protection en y ajoutant une dimension égalitaire.

Si la jurisprudence ainsi que la doctrine québécoises ont pris acte de cette caractéristique fondamentale ${ }^{8}$, contribuant par le fait même à la compréhension de l'article 10, aucun auteur, à notre connaissance, n'a proposé d'étude approfondie sur ses contours exacts et sa portée réelle. Telle est la tâche à laquelle nous entendons nous livrer.

8. Supra notes 5 et 6 . La Cour européenne des droits de l'homme ainsi que la doctrine ont également reconnu cet aspect «fondateur» de l'article 14 de la Convention européenne, infra note 9 : Cha'are Shalom Ve Tsedek c. France, arrêt du 27 juin 2000 au para. 86 (ci-après Cha'are Shalom Ve Tsedek); Thlimmenos c. Grèce, arrêt du 6 avril 2000 au para. 40 (ciaprès Thlimmenos); Rekvényi c. Hongrie, arrêt du 20 mai 1999 au para. 67 (ci-après Rekvényi); Botta c. Italie, arrêt du 24 février 1998 au para. 39 (ci-après Botta); Van Raalte c. Pays-Bas, arrêt du 21 février 1997 au para. 33 (ci-après Van Raalte); Gaygusuz c. Autriche, arrêt du 16 septembre 1996 au para. 36 (ci-après Gaygusuz); Karlheinz Schmidt c. Allemagne, arrêt du 18 juillet 1994 au para. 22 (ci-après Karlheinz Schmidt); Inze, arrêt du 28 octobre 1987 au para. 36 (ci-après Inze); Abdulaziz, Cabales et Balkandali c. RoyameUni, arrêt du 28 mai 1985 au para. 71 (ci-après Abdulaziz); X et Y c. Pays-Bas, arrêt du 26 mars 1985 au para. 32 (ci-après $X$ et $Y$ ); Rasmussen c. Danemark, arrêt du 28 novembre 1984 au para. 29 (ci-après Rasmussen); Van der Mussele, arrêt du 22 novembre 1983 au para. 43 (ci-après Van der Mussele); Affaire «relative à certains aspects du régime linguistique de l'enseignement en Belgique», arrêt du 23 juillet 1968 à la p. 38 (ci-après l'Affaire linguistique belge); Airey, arrêt du 9 octobre 1979 au para. 30 (ci-après Airey); Marckx c. Belgique, arrêt du 13 juin 1979; Syndicat national de la police belge, arrêt du 27 octobre 1975 au para. 44 (ci-après Syndicat national de la police belge); M.-A. Eissen, «L' «autonomie» de l'article 14 de la Convention européenne des droits de l'homme dans la jurisprudence de la Commission», dans Mélanges offerts à Polys Modinos, Paris, Éditions A. Pedone, 1968, 122 à la p. 123; J. E.S. Fawcett, The Application of the European Convention on Human Rights, Oxford, Oxford University Press, 1987, 295; D. Gomien, D. Harris et L. Zwaak, Convention européenne des Droits de l'Homme et la Charte sociale européenne : droit et pratique, Strasbourg, Les Éditions du Conseil de l'Europe, 1997, 371; J.-P. Jacqué, «Le principe d'égalité» dans G.-A. Beaudoin, dir., Vues canadiennes et européennes des droits et libertés, Actes des Journées Stratsbourgeoises, Cowansville (Qc), Yvon Blais, 1988, 77 à la p. 81; P. Lambert, «Vers une évolution de l'interprétation de l'article 14 de la Convention européenne des droits de l'homme ?» (1998) Rev. trim. dr. h. 497; L.-E. Pettiti, E. Decaux et P.-H. Imbert, La Convention européenne des droits de l'homme - Commentaire article par article, $2^{\mathrm{e}}$ éd., Paris, Éditions Economica, 1999, 478; F. Sudre, Droit international et européen des droits de l'homme, $4^{\mathrm{e}}$ éd., Paris, Éditions Presses universitaires de France, 1999, 305; K. Vasak, La Convention européenne des droits de l'homme, Strasbourg, Bibliothèque constitutionnelle et de science politique, 1964. 
Il sera intéressant à cet égard de s'inspirer et de tirer profit de la jurisprudence à jour de la Cour européenne des droits de l'homme sur l'article 14 de la Convention de sauvegarde des droits de l'homme et des libertés fondamentales $^{9}$, signée à Rome le 4 novembre 1950 quelque 25 ans avant l'entrée en vigueur de la Charte québécoise ${ }^{10}$. La facture de cette disposition antidiscriminatoire européenne est loin d'être étrangère à celle de l'article 10, ce qui n'est pas étonnant puisque notre législateur s'en est inspiré dans l'adoption de la norme québécoise d'égalité ${ }^{11}$. L'article 14 prévoit ainsi que :

La jouissance des droits et libertés reconnus dans la présente Convention doit être assurée, sans distinction aucune, fondée notamment sur le sexe, la race, la couleur, la langue, la religion, les opinions politiques ou toutes autres opinions, l'origine nationale ou sociale, l'appartenance à une minorité nationale, la fortune, la naissance ou toute autre situation.

Le Tribunal des droits de la personne du Québec, forum spécialisé en la matière, de même que plusieurs auteurs ont d'ailleurs reconnu le lien de parenté direct qu'entretiennent les normes d'égalité québécoise et européenne ${ }^{12}$. Comme l'article 10, l'article 14 constitue donc une modalité de particularisation d'autres droits et libertés. Voilà donc l'occasion d'expliciter plus en détails les

9. $\quad$ (1955) 213 R.T.N.U. 221, S.T.E. $\mathrm{n}^{\circ} 5$ (ci-après Convention).

10. Les auteurs W. A. Schabas et D. Turp, Droit international, canadien et québécois des droits et libertés : notes et documents, $2^{\mathrm{e}}$ éd., Cowansville (Qc), Yvon Blais, 1998 à la p. 195, affirment en effet que «[...] la jurisprudence de [...] la Cour européenne des droits de l'homme est d'un intérêt très particulier pour les juristes canadiens et québécois».

11. Ibid.

12. Bertrand, supra note 5 à la p. 1690; Commission des droits de la personne et des droits de la jeunesse c. Quévillon, [1999] J.L. 193 à la p. 197 (T.D.P.Q.) (ci-après Quévillon); Commission des droits de la personne c. Centre d'accueil Villa Plaisance, [1996] R.J.Q. 511 à la p. 527 (T.D.P.Q.) (ci-après Villa Plaisance); Commission des droits de la personne du Québec c. Immeubles Ni/Dia, [1992] R.J.Q. 2977 à la p. 2984 (T.D.P.Q.); Commission des droits de la personne du Québec c. Commission scolaire Saint-Jean-sur-Richelieu, [1991] R.J.Q. 3003 à la p. 3025 (T.D.P.Q.); P. Blache, supra note 3 à la p. 110; P. Carignan, supra note 3 à la p. 527; M. Caron, «Le droit international des droits de la personne : son application au Québec par la Commission et le Tribunal des droits de la personne» (19891990) 6 R.Q.D.I. 104 à la p. 108; M. Caron, supra note 3 à la p. 43; D. Proulx, «La norme québécoise d'égalité dérape en Cour suprême : commentaire des arrêts Forget, Devine et Ford» (1990) 24 R.J.T. 375 aux pp. 393 et 394; D. Proulx, supra note 3 aux pp. 499 et 500. 
Non-indépendance et autonomie de la norme
(2004) 35 R.D.U.S. d'égalité québécoise : des concepts «fondateurs»
qui méritent d'être mieux connus

conséquences pratiques de cette réalité commune aux normes nonindépendantes mais autonomes d'égalité québécoise et européenne.

Notre cheminement nous mènera inévitablement à l'analyse de la jurisprudence récente de la Cour d'appel sur l'article 10, laquelle s'inspire largement des développements dont a fait l'objet l'article 15 de la Charte canadienne des droits et libertés et qui ont fait de la dignité humaine le cœur de la garantie constitutionnelle d'égalité. Nous constaterons que la voie empruntée par la Cour suprême ainsi que par la Cour d'appel n'est pas sans créer des difficultés d'interprétation d'une importance telle que le sens de l'article 10 dans la Charte en est désormais compromis.

\section{La non-indépendance de l'article 10 de la Charte : aux frontières de la norme d'égalité québécoise}

En raison de son champ d'application limité, le droit à la nondiscrimination ne peut servir de base unique à un recours et doit nécessairement être invoqué en combinaison ou en corrélation avec un autre droit ou liberté de la personne ${ }^{13}$. Les propos de l'auteur Marc Bossuyt, qui exprime très bien cette réalité en ce qui concerne l'article 14 de la Convention européenne, s'appliquent sans réserve à l'article 10 de la Charte :

La discrimination est interdite pour tous les droits mais elle ne l'est pas, quand elle touche des intérêts non protégés par le droit. Même un traitement discriminatoire dont le motif est généralement condamné (tel la race) ne peut être juridiquement interdit, si ce traitement ne concerne pas un droit subjectif reconnu. [...] Il y a complète simultanéité entre l'octroi de protection juridique à un certain intérêt et l'interdiction de tout traitement arbitraire dans la jouissance de cet intérêt. La discrimination devient interdite au moment même où l'intérêt reçoit la protection de la loi. Le caractère juridique de l'intérêt en jeu apparaît ainsi comme la condition d'applicabilité de la règle de non-discrimination. ${ }^{14}$

13. Bertrand, supra note 5 à la p. 1690; Bouffard, supra note 5 au para. 11; Quévillon, ibid. à la p. 197.

14. M. Bossuyt, L'interdiction de la discrimination dans le droit international des droits de l’homme, Bruxelles, Éditions Bruylant, 1976 aux pp. 74 et 75. Voir également : G. CohenJonathan, La Convention européenne des droits de l'homme, Paris, Éditions Presses 

qui méritent d'être mieux connus

Autrement dit, la norme d'égalité québécoise n'est donc pas susceptible d'application indépendante ${ }^{15}$. S'il y a unanimité de principe à cet égard, tant en jurisprudence qu'en doctrine, on parle toutefois généralement d'absence «d'autonomie» ${ }^{16}$ pour illustrer cette caractéristique du droit à la nondiscrimination. Ainsi, comme la Charte «ne le reconnaît qu'à l'égard des droits et libertés de la personne [...] [,] [il] n'est pas envisagé comme un droit autonome [...]». ${ }^{17} \mathrm{La}$ Cour européenne des droits de l'homme fait cependant clairement la distinction entre l'absence d'application indépendante de l'article 14 et sa portée autonome. ${ }^{18}$ Au Québec, le professeur Daniel Proulx est

Universitaires d'Aix-Marseille et Economica, 1989 aux pp. 538 et 539; D. Gomien, D. Harris et L. Zwaak, supra note 8 aux pp. 371 et 372; M.-A. Eissen, supra note 8 à la p. 123; J.E.S. Fawcett, supra note 8 à la p. 295; M. Levinet, «La juridicité problématique du droit au développement de la personne humaine dans la jurisprudence récente des organes de la Convention européenne des droits de l'homme» dans J.-Y. Morin et G. Otis, dir., Les défis des droits fondamentaux, Actes des deuxièmes Journées scientifiques du Réseau Droits fondamentaux de l'Agence Universitaire de la Francophonie, Bruxelles, Éditions Bruylant, 2000, 95 à la p. 116; L.-E. Pettiti, E. Decaux et P.-H. Imbert, supra note 8 à la p. 478; F. Sudre, supra note 8 à la p. 305; F. Sudre, «La portée du droit à la non-discrimination : de l'avis de l'Assemblée du Conseil d'État du 15 avril 1996, Mme Doukouré, à l'arrêt de la Cour européenne des droits de l'homme du 16 septembre 1996, Gaygusuz c/ Autriche» (1997) Rev. fr. dr. adm. 966, à la p. 968.

15. Villa Plaisance, supra note 12 à la p. 527; D. Proulx, supra note 3 aux pp. 488 et 489.

16. Gosselin c. Québec (Procureur général), supra note 6 aux para. 429 et 430 (J. Lebel); Ruel c. Marois, [2001] R.J.Q. 2590 au para. 129 (C.A.); Gosselin c. Québec (Procureur général), [2002] R.J.Q. 1298 à la p. 1306 (C.A.) (ci-après Gosselin); Habachi, supra note 5 à la p. 2537; Commission scolaire St-Jean-sur-Richelieu, supra note 5 à la p. 1243; Québec (Ville) c. Commission des droits de la personne du Québec, supra note 6 à la p. 844; Godin c. Société de l'assurance automobile du Québec, C.S., Montréal, 500-05-051339-990, 200005-16; Poulin, supra note 5 à la p. 8 du texte intégral; Bouffard, supra note 5 au para. 11; Quévillon, supra note 12 à la p. 197; H. Brun et G. Tremblay, supra note 5 à la p. 1106; P. Carignan, supra note 3 à la p. 527; L. Martineau, «Le statut d'emploi peut-il constituer un motif de distinction illicite en vertu du paragraphe 15 (1) de la Charte canadienne et de l'article 10 de la Charte québécoise ?» dans Service de la formation permanente, Barreau du Québec, Développements récents en droit administratif (1994), Cowansville (Qc), Yvon Blais, 127 à la p. 140; H. Tessier, «Les articles 12 et 13 de la Charte, ces méconnus : quand le droit privé rencontre le droit public», dans Service de la formation permanente, Barreau du Québec, Les 25 ans de la Charte québécoise, Cowansville (Qc), Yvon Blais, 2000, 119 à la p. 122.

17. Commission scolaire St-Jean-sur-Richelieu, supra note 5 à la p. 1243.

18. Voir la jurisprudence à laquelle il est fait référence à la note 8. La Cour européenne ainsi que la doctrine parlent d'absence «d'existence» indépendante de l'article 14. Il s'agit d'un terme qui, à notre avis, n'exprime pas de façon rigoureusement exacte cette particularité des 
pratiquement le seul ${ }^{19}$ à faire cette distinction qui n'est pas sans importance puisqu'il s'agit de deux aspects différents de l'application de l'article $10^{20}$.

L'absence d'application indépendante de la norme d'égalité québécoise engendre des conséquences pratiques importantes. Elles sont au nombre de trois. Premièrement, comme nous l'avons vu, elle ne peut être invoquée seule sans rattachement à une autre disposition, si bien que lorsque aucun autre droit ou liberté n'est a priori applicable, elle ne peut tout simplement pas entrer en jeu aussi moralement condamnable que puisse être une distinction ${ }^{21}$.

Deuxièmement, lorsqu'il y a violation en soi d'un droit ou d'une liberté, il n'est pas nécessaire pour le tribunal d'analyser le litige sous un angle égalitaire, du moins techniquement ${ }^{22}$. Comme il y a déjà violation du droit invoqué à titre principal, la constatation d'une discrimination dans la reconnaissance ou l'exercice de celui-ci est susceptible d'être considérée comme

normes d'égalité québécoise et européenne. Le droit à l'égalité possède une existence propre et distincte des autres droits. Il ne peut cependant s'appliquer seul ou de façon indépendante, c'est-à-dire sans rattachement à l'un de ces droits.

19. Mise à part l'Honorable Michèle Rivet du Tribunal des droits de la personne dans la décision Villa Plaisance, supra note 12 à la p. 527. Madame la juge a toutefois abandonné cette expression pour utiliser plutôt le mot «autonome» comme le démontre les décisions récentes Bouffard, supra note 5 au para. 11 et Quévillon, supra note 12 à la p. 197.

20. D. Proulx, supra note 3 à la p. 489 (note 357); D. Proulx, supra note 6 à la p. 518.

21. Morand c. Québec (Procureur général), C.A., Montréal, 500-09-001436-914, 2000-10-03, AZ-50079307 au para. 7; Lavigne c. Québec (Attorney General), C.S., Montréal, 500-05056434-002, 2000-12-14, AZ-50081709 (ci-après Lavigne); Syndicat étudiant du cégep Marie-Victorin c. Collège d'enseignement général et professionnel Marie-Victorin, C.S., Montréal, 500-05-050773-991, 2000-05-04, AZ-00021577 (ci-après Collège MarieVictorin); Compagnie d'assurance Guardian du Canada c. Leblanc, [1999] R.R.A. 670, 678 à 680 (C.S.); Wagner c. I.N.G., Le groupe Commerce, compagnie d'assurance, [2001] R.R.A. 531, 533 (C.Q.) (ci-après Wagner); D. Proulx, supra note 3 à la p. 489. Pour l'article 14 de la Convention voir : Botta, supra note 8 au para. 39.

22. Bien entendu, la discrimination dans la reconnaissance ou l'exercice d'un droit est autant déplorable que la violation même ou isolée de ce droit. Cependant, dans une perspective strictement juridique ou technique, fondée sur la dépendance de l'article 10 aux autres dispositions de la Charte, il n'est pas absolument nécessaire pour un tribunal qui a déjà constaté une atteinte à un autre droit de vérifier si le plaignant a aussi été victime de discrimination dans son exercice. 
une circonstance aggravante ${ }^{23}$ et la norme d'égalité ne joue alors qu'un rôle subsidiaire $^{24}$. L'atteinte même d'un droit ou d'une liberté pourra d'ailleurs emporter, dans certains cas, ${ }^{25}$ celle de la norme d'égalité ${ }^{26}$ puisque la personne ainsi privée du droit en question est susceptible de subir, par le fait même, une distinction par rapport aux personnes dont les droits sont respectés.

C'était le cas dans l'arrêt Ford c. Québec (Procureur général) de la Cour suprême. ${ }^{27}$ Il s'agissait dans cette affaire de savoir si les articles 58 et 69 de la Charte de la langue française du Québec respectaient la liberté d'expression protégée par l'article 3 de la Charte québécoise. ${ }^{28}$ La Cour devait également déterminer si ces dispositions contrevenaient au droit à l'égalité dans l'exercice de la liberté d'expression, contrairement aux articles 3 et 10 de la Charte. Après avoir conclu que la liberté d'expression comprend la liberté de s'exprimer dans la langue de son choix et qu'elle ne se limite pas à l'expression politique mais englobe aussi l'expression commerciale, la Cour a jugé que les articles 58 et 69 portaient atteinte de façon injustifiable à l'article $3 .{ }^{29}$

23. La dimension égalitaire d'un recours peut toutefois s'avérer fort importante pour le demandeur en vertu de l'article 10 de la Charte. En effet, en invoquant qu'il est victime non seulement d'une violation d'un droit ou d'une liberté, mais aussi de discrimination dans la reconnaissance ou l'exercice de ce droit, il peut porter plainte devant la Commission des droits de la personne et des droits de la jeunesse. Mentionnons enfin qu'en vertu du paragraphe 134(1) et de l'alinéa 136(1) de la Charte, la discrimination constitue une infraction, son auteur pouvant d'ailleurs faire l'objet d'une poursuite pénale par la Commission.

24. J.-P. Jacqué, supra note 8 à la p. 82 .

25. Encore faut-il que la distinction soit fondée sur un motif énuméré.

26. M. Bossuyt, supra note 14 à la p. 232.

27. Ford c. Québec (Procureur général), [1988] 2 R.C.S. 712 (ci-après Ford). Pour des exemples en vertu de l'article 14 de la Convention, voir : Ex-Roi de Grèce et autres $\mathrm{c}$. Grèce, arrêt du 23 novembre 2000; Kiliç c. Turquie, arrêt du 28 mars 2000; Beyeler c. Italie, arrêt du 5 janvier 2000; Willie c. Liechtenstein, arrêt du 28 octobre 1999; Arslan c. Turquie, arrêt du 8 juillet 1999; Okçuoglu c. Turquie, arrêt du 8 juillet 1999; Chassagnou et autres c. France, arrêt du 29 avril 1999; Yasa c. Turquie, arrêt du 2 septembre 1998; Keegan c. Irlande, arrêt du 26 mai 1994; X et Y, supra note 8; Airey, supra note 8.

28. Charte de la langue française, L.R.Q. c. C-11. Les articles 58 et 69 exigeaient que l'affichage public et la publicité commerciale se fassent seulement en français et que seule soit utilisée, par une compagnie, sa raison sociale en français. L'article 3 de la Charte énonce quant à lui que : «Toute personne est titulaire des libertés fondamentales telles [...] la liberté d'expression [...].»

29. $\quad$ Ford, supra note 27 à la p. 767. 
Étant donné ce constat de violation injustifiée de la liberté d'expression, il n'était alors plus nécessaire pour le plus haut tribunal du pays de vérifier si dans l'exercice de cette liberté il y avait eu discrimination. ${ }^{30} \mathrm{~S}$ ' agissant toutefois d'un aspect important du litige, la Cour suprême, comme le fait d'ailleurs la Cour européenne, ${ }^{31}$ décida tout de même de répondre à cette question, ${ }^{32}$ se montrant ainsi soucieuse d'accorder au principe d'égalité la place centrale qui lui revient dans la Charte. ${ }^{33}$ Mais comme on pouvait s'y attendre, la violation de la liberté d'expression, liée à l'expression linguistique, emportait aussi la violation du droit à l'exercice de cette liberté sans discrimination fondée sur la langue :

Le droit ou la liberté de la personne en cause est la liberté de s'exprimer dans la langue de son choix qui [...] est reconnue par l'art. 3 de la Charte québécoise. En l'espèce, la restriction imposée à ce droit n'était pas justifiable en vertu de l'article 9.1 de la Charte québécoise. La distinction fondée sur la langue usuelle créé par l'art. 58 de la Charte de la langue française a donc pour effet de détruire le droit à la reconnaissance et à l'exercice, en pleine égalité, de cette liberté. ${ }^{34}$

Il existe une troisième conséquence pratique de l'absence d'application indépendante de la norme d'égalité québécoise. Il s'agit du cas où il y a effectivement violation d'un droit ou d'une liberté de la personne mais que celle-ci est justifiée. Dans ce contexte, l'analyse d'une atteinte au droit à l'égalité est à notre avis impossible. En effet, comme l'application de l'article 10 est intrinsèquement dépendante de celle d'une autre disposition, la violation justifiée de cette dernière a nécessairement pour effet d'empêcher l'entrée en scène de la norme d'égalité. Une personne dont la restriction à la reconnaissance

30. C'est d'ailleurs le constat que tire la Cour, supra note 27 à la p.781 : «Compte tenu de la conclusion qui précède, il n'est pas nécessaire en l'espèce que la Cour se prononce sur l'argument des intimées suivant lequel les art. 58 et 69 de la Charte de la langue française sont inopérants parce qu'ils violent la garantie contre la discrimination fondée sur la langue, énoncée à l'art. 10 de la Charte des droits et libertés de la personne du Québec.»

31. Airey, supra note 8 au para. 30 et $X$ et $Y$, supra note 8 au para. 32.

32. Ford, supra note 27 à la p. 781.

33. Voir, à propos de l'article 14 : J.-F. Flauss, «Discrimination positive et Convention européenne des droits de l'homme», dans Pouvoir et libertés - Études offertes à Jacques Mourgeon, Bruxelles, Les Éditions Bruylant, 1998, 415 à la p. 416.

34. $\quad$ Ford, supra note 27 à la p. 787. 
ou l'exercice d'un droit a été jugée justifiée ne peut tout simplement pas se plaindre de discrimination dans la reconnaissance ou l'exercice de ce droit si cette dernière allégation repose sur les mêmes faits ou circonstances. ${ }^{35}$

Ainsi, dans l'arrêt Devine c. Québec (Procureur général), ${ }^{36}$ la Cour suprême devait déterminer si les articles 52 et 57 de la Charte de la langue française respectaient la liberté d'expression garantie par l'article 3 de la Charte québécoise. $^{37}$ L'article 52 prévoyait que les catalogues, les brochures, les dépliants et toute autre publication de même nature devaient être rédigés en français. Pour sa part, l'article 57 énonçait la même règle en ce qui concerne les formulaires de demande d'emploi, les bons de commande, les factures, les reçus et les quittances. Cependant, en vertu de l'article 89, lorsque l'usage exclusif du français n'était pas exigé par une disposition de la loi (c'était le cas des articles 52 et 57), il était aussi permis d'utiliser une autre langue.

À la lumière de l'arrêt Ford, la Cour a conclu que cette restriction à la liberté d'expression portait atteinte à l'article $3 .{ }^{38}$ En revanche, dans la mesure où les articles 52 et 57 permettaient l'usage concurrent d'une autre langue, cette violation était justifiée en vertu de l'article 9.1 de la Charte. ${ }^{39}$ Par conséquent, comme l'article 10 ne possède pas d'application indépendante, la justification de la violation de l'article 3 rendait impossible l'analyse d'une atteinte à la norme d'égalité. C'est, selon nous, ce que la Cour suprême a voulu expliquer en ces termes :

Chaque fois qu'il est allégué qu'une distinction fondée sur un motif interdit par l'art. 10 a pour effet de compromettre ou de détruire un droit que prévoit l'article 3, la portée de cet article doit être déterminée à la lumière de l'art. 9.1. Lorsque, comme en l'espèce, l'art. 9.1 a pour effet de

35. Devine c. Québec (Procureur général), [1988] 2 R.C.S. 790 à la p. 818 (ci-après Devine) et Syndicat Northcrest c. Amselem, [2002] R.J.Q. 906 aux pp. 922 et 923 (C.A.) (J. Morin) (ci-après Amselem). Pour l'article 14 de la Convention, voir : Revényi, supra note 8 au para. 68; Chapman c. Royaume-Uni, arrêt du 18 janvier 2001 au para. 129; Coster c. RoyaumeUni, arrêt du 18 janvier 2001 au para. 141 (ci-après Coster).

36. Supra note 35.

37. $\quad$ Charte de la langue française, supra note 28.

38. Devine, supra note 35 à la p. 813.

39. Ibid. 
limiter la portée de la liberté d'expression que garantit l'art. 3, l'art. 10 ne peut être invoqué pour contourner les limites raisonnables à cette liberté et y substituer une garantie absolue de liberté d'expression. ${ }^{40}$

En effet, comme le traitement égalitaire ne peut être revendiqué qu'à l'égard de la reconnaissance ou l'exercice de la liberté d'expression, il en résulte qu'une restriction justifiée selon l'article 9.1 à la reconnaissance ou l'exercice même de cette liberté empêche logiquement la revendication du droit à la nondiscrimination. ${ }^{41}$

Voilà donc les trois grandes conséquences qu'emporte l'absence d'application indépendante de la norme d'égalité québécoise lorsque celle-ci, il importe de le préciser, est invoquée en corrélation avec un droit ou une liberté fondamental, ${ }^{42}$ un droit politique, ${ }^{43}$ un droit judiciaire ou un droit économique, social ou culturel. ${ }^{44}$

Tel n'est pas le cas, en effet, lorsque la discrimination se pratique dans l'un des domaines d'activités protégés aux articles 11 à 18 ou l'article 19. Ces dernières dispositions se trouvent au Chapitre 1.1 de la Charte, lui-même compris dans la Partie I intitulée «Les droits et libertés de la personne». ${ }^{45}$ Elles ne consacrent cependant, en elles-mêmes, aucun droit distinct du droit à l'égalité. ${ }^{46}$ Elles visent plutôt à indiquer que dans certains domaines spécifiques d'activités comme la publicité, ${ }^{47}$ la formation et le contenu d'actes juridiques, ${ }^{48}$

40. $\quad$ Ibid. à la p. 818.

41. Voir aussi : Amselem, supra note 35 aux pp. 922 et 923 (J. Morin).

42. Charte, supra note 1 aux art. 1 à 9 .

43. Ibid. aux art. 21 et 22 .

44. Ibid. aux art. 23 à 38 et 39 à 48 .

45. Nos italiques.

46. H. Brun et G. Tremblay, supra note 5 à la p. 1107; D. Proulx, supra note 3 à la p. 481 .

47. Charte, supra note 1 à l'art. 11.

48. Ibid. aux art. 12 et 13. 

qui méritent d'être mieux connus

l'accès à des lieux publics ${ }^{49}$ et en matière d'emploi,${ }^{50}$ la discrimination telle que définie à l'article $10^{51}$ est interdite. ${ }^{52}$

Les articles 11 à 18 et l'article 19 sont par conséquent intrinsèquement liés à la norme d'égalité et ne peuvent être invoqués seuls, c'est-à-dire indépendamment de celle-ci. ${ }^{53}$ En effet, comme ils n'énoncent pas de droits en soi, ils doivent nécessairement s'appliquer en interaction avec l'article 10 et en constituent donc une contextualisation. ${ }^{54}$ Chacun a ainsi le droit, par exemple, de ne pas faire l'objet d'une distinction, exclusion ou préférence, fondée sur un

49. Ibid. à l'art. 15 .

50. Ibid. aux art. 16, 17, 18 et 19 .

51. Tremblay c. Syndicat des employées et employés professionnels et de bureau, section locale 57, [1996] R.J.Q. 1850 à la p. 1859 (C.S.) (ci-après Tremblay), infirmé par Syndicat des employées et employés professionnels et de bureau, section locale 57 c. Tremblay, [2000] R.J.Q. 1098 (C.A.), mais pour d'autres raisons. La Cour suprême a confirmé l'arrêt de la Cour d'appel : Tremblay c. Syndicat des employées et employés professionnels et de bureau, section locale 57, 2002 CSC 44, J.E. 2002-813. En première instance, la Cour supérieure avait limité ses motifs aux articles 13, 16 et 19, mais, à notre avis, le même raisonnement s'applique aux articles 11, 12, 15, 17 et 18 de la Charte. Voir aussi : Québec (Ville) c. Commission des droits de la personne du Québec, supra note 6 à la p. 839; Commission des droits de la personne du Québec c. Collège Mérici, [1990] R.J.Q. 604 à la p. 607 (C.Q.); Commission des droits de la personne et des droits de la jeunesse c. Nicolet (Ville de), T.D.P.Q., Trois-Rivières, 400-53-000001-009, 2001-07-25, AZ-50099107 aux para. 65 et 66; Commission des droits de la personne et des droits de la jeunesse c. Institut Demers Inc., [1999] R.J.Q. 3101 à la p. 3109 (T.D.P.Q.); D. Proulx, supra note 3 à la p. 481.

52. L'article 12 énonce par exemple que : «[Discrimination dans la formation d'acte juridique] Nul ne peut, par discrimination, refuser de conclure un acte juridique ayant pour objet des biens ou des services ordinairement offerts au public». (nos italiques)

53. Les articles 18.1 et 18.2 ne font pas référence à la «discrimination» que définit l'article 10 . Il s'ensuit que ces deux dispositions sont susceptibles de s'appliquer seules, indépendamment de la norme d'égalité. Le libellé des articles en question appui d'ailleurs ce constat : «[Renseignements relatifs à un emploi] 18.1. Nul ne peut, dans un formulaire de demande d'emploi ou lors d'une entrevue relative à un emploi, requérir d'une personne des renseignements sur les motifs visés dans l'article 10 sauf si ces renseignements sont utiles à l'application de l'article 20 ou à l'application d'un programme d'accès à l'égalité existant au moment de la demande.» «[Culpabilité à une infraction] 18.2. Nul ne peut congédier, refuser d'embaucher ou autrement pénaliser dans le cadre de son emploi une personne du seul fait qu'elle a été déclarée coupable d'une infraction pénale ou criminelle, si cette infraction n'a aucun lien avec l'emploi ou si cette personne en a obtenu le pardon.». Les articles 18.1 et 18.2 interdisent ainsi carrément certains comportements qui ne sont justifiables qu'aux conditions prévues.

54. Voir H. Brun et G. Tremblay, supra note 5 à la p. 1107. 
motif énuméré qui a pour effet de détruire ou de compromettre le droit à l'égalité dans l'accessibilité à un lieu public. ${ }^{55}$

Or, comme on peut le constater, il ne s'agit plus de vérifier dans la reconnaissance ou l'exercice de quel droit ou liberté il y a discrimination, mais bien dans quel domaine d'activités. L'article 10, lorsque combiné à l'un des articles 11 à 18 ou l'article 19, constitue par conséquent une norme d'égalité indépendante des autres droits et libertés de la personne. Un lien entre le droit à l'égalité et un domaine d'activités protégé par la Charte est ainsi suffisant ${ }^{56}$ et il n'est pas nécessaire d'identifier, en plus, un droit ou une liberté de la personne dans lequel se pratique la distinction. ${ }^{57}$

\section{A. L'application large et libérale de la norme d'égalité pour pallier sa limitation inhérente de non-indépendance}

Sous réserve de ce qui vient d'être dit au sujet des articles 11 à 18 et 19, un lien doit donc nécessairement unir l'article 10 à un autre droit ou liberté de la personne pour qu'il puisse s'appliquer. Mais quelle doit en être l'intensité ? Doit-il s'agir d'un lien étroit ou plus large ? En ce qui concerne l'article 14 de la Convention, un auteur avance que la discrimination alléguée doit concerner des droits «ayant un lien étroit avec un de ceux expressément reconnus dans la Convention [...]». ${ }^{58}$ Il s'agit là, cependant, d'une interprétation stricte que n'ont retenue ni la Cour européenne ni la Cour d'appel du Québec.

La Cour européenne interprète en effet de façon large et libérale le lien devant unir l'article 14 à un droit ou une liberté reconnu dans la Convention. Il suffit ainsi que les faits du litige tombent sous l'empire, dans le domaine, le cadre ou les modalités d'au moins une disposition de la Convention pour paver

55. Charte, supra note 1 aux art. 10 et 15 .

56. Les propos suivants du professeur Proulx semblent indiquer qu'il est également de cette opinion : «Il faut $[\ldots]$ pour conclure à discrimination ou inégalité, rattacher une distinction fondée sur un motif discriminatoire à un droit, liberté ou domaine d'activité reconnu par la Charte [...]» (Nous soulignons) : D. Proulx, supra note 3 à la p. 489.

57. Les professeurs Brun et Tremblay ne partagent pas ce raisonnement et semblent plutôt d'avis que l'application de l'article 10, même dans ce contexte, implique l'identification d'un autre droit ou liberté : H. Brun et G. Tremblay, supra note 5 à la p. 1107.

58. P. Lambert, supra note 8 à la p. 501. 
la voie à l'application de l'article $14 .^{59}$ Il en est de même pour l'article 10 de la Charte, tel que le reconnaissait la Cour d'appel en 1994 dans l'arrêt Commission scolaire St-Jean-Sur-Richelieu. ${ }^{60}$ Un rapport étroit entre l'allégation de discrimination et ce qui est impliqué par une autre disposition n'est donc pas nécessaire ${ }^{61}$ comme le démontre l'analyse de certains arrêts de principe rendus par la Cour européenne dont il convient de prendre connaissance. Cela aura l'avantage d'illustrer comment le concept théorique invoqué ci-avant s'incarne dans la pratique par sa mise en application jurisprudentielle.

Dans l'affaire Syndicat national de la police belge, ${ }^{62}$ par exemple, le syndicat requérant contestait, sur la base des articles 11 (droit d'association) ${ }^{63}$ et 14 de la Convention, la validité de dispositions législatives qui accordaient à certains syndicats le droit d'être consultés par l'État et le refusaient à d'autres selon les critères établis.

La Cour a conclu que le droit d'association du requérant avait été respecté par l'État belge dans la mesure où ce dernier n'avait pas empêché le syndicat

59. Thlimmenos, supra note 8 au para. 40; Botta, supra note 8 au para. 39; Van Raalte, supra note 8 au para. 33; Gaygusuz, supra note 8 au para. 36; Karlheinz Schmidt, supra note 8 au para. 22; Inze, supra note 8 au para. 36; Abdulaziz, supra note 8 au para. 71; Rasmussen, supra note 8 au para. 29; Van der Mussele, supra note 8 au para. 43; Syndicat national de la police belge, supra note 8 au para. 45 .

60. Commission scolaire St-Jean-sur-Richelieu, supra note 5 à la 1243. Voir aussi : Poulin, supra note 5 à la p. 8 du texte intégral; Bertrand, supra note 5 aux pp. 1690 et 1691; P. Bosset, «Les droits économiques et sociaux : parents pauvres de la Charte québécoise ?» (1996) 75 R. du B. Can. 583 aux pp. 592 et 593; H. Brun et G. Tremblay, supra note 5 à la p. 1106; P. Carignan, supra note 3 aux pp. 527 et 530; M. Caron, supra note 3 à la p. 52; D. Proulx, supra note 3 à la p. 497 et s.

61. J.-P. Jacqué, supra note 8 à la p. 82; G. Cohen-Jonathan, supra note 14 aux pp. 538 et 539 ; M. Levinet, supra note 14 aux pp. 116 et 117; L.-E. Pettiti, E. Decaux et P.-H. Imbert, supra note 8 à la p. 479. On pourrait penser qu'une telle application des normes québécoise et européenne d'égalité va à l'encontre du libellé des articles 10 de la Charte et 14 de la Convention, dénaturant ainsi ce que le législateur avait prévu en limitant leur application aux autres droits et libertés. Il s'agirait toutefois, selon nous, d'une approche réductrice de l'applicabilité et du rôle de la norme d'égalité dans la Charte ou la Convention que n'ont heureusement retenu ni la Cour européenne ni la Cour d'appel du Québec.

62. Supra note 8.

63. Le premier alinéa de l'article 11 prévoit que : «Toute personne a droit à la liberté de réunion pacifique et à la liberté d'association, y compris le droit de fonder avec d'autres des syndicats et de s'affilier à des syndicats pour la défense de ses intérêts.» 
exclu de la procédure de consultation d'être entendu, d'exprimer ses idées et de faire valoir ses intérêts par d'autres moyens. Autrement dit, l'article 11 protège le droit d'être entendu mais ne garantit pas les moyens particuliers par lesquels un syndicat peut effectivement exercer le droit qu'il énonce. ${ }^{64}$

Si l'article 11 n'assure pas le droit d'être directement consulté par l'État selon une procédure mise de l'avant par une loi, il n'en demeure pas moins que la consultation constitue une façon d'exercer le droit d'association consacré et, par le fait même, en est une modalité, ce qu'a d'ailleurs constaté la Cour avec raison. ${ }^{65}$ L'article 14 se trouvait dès lors applicable. ${ }^{66}$

L'arrêt Petrovic c. Autriche illustre aussi très bien la souplesse interprétative ou le dynamisme de la Cour européenne dans l'application de l'article $14 .{ }^{67}$ Dans cette affaire, le requérant contestait, en vertu des articles 8 (respect de la vie privée et familiale) ${ }^{68}$ et 14 de la Convention, la décision d'une instance administrative autrichienne qui avait refusé sa demande d'allocation pour congé parental au motif que la loi prévoyait que seules les mères pouvaient en bénéficier. ${ }^{69}$

N'imposant à l'État que l'obligation dite «négative» ${ }^{70}$ de respecter la vie privée et familiale de ses ressortissants et ne l'obligeant pas à mettre en place des mesures destinées à faciliter l'exercice du droit qu'il consacre, l'article 8 n'avait pas été violé dans les circonstances.

Cependant, dans la mesure où le versement d'une allocation visait à favoriser la vie familiale, la mesure adoptée par l'État avait une incidence sur l'organisation de celle-ci et, donc, sur la jouissance du droit énoncé à l'article 8.

64. Syndicat national de la police belge, supra note 8 aux para. 38 à 40 .

65. Ibid. au para. 45.

66. En l'espèce, toutefois, la Cour a jugé que la distinction subie par le syndicat requérant était justifiée dans les circonstances : Ibid. au para. 48.

67. Petrovic c. Autriche, arrêt du 27 mars 1998.

68. Le premier paragraphe de l'article 8 énonce que : «Toute personne a droit au respect de sa vie privée et familiale, de son domicile et de sa correspondance.»

69. Petrovic c. Autriche, supra note 67 au para. 9.

70. Par opposition aux obligations dites «positives» qui nécessitent une intervention de l'État et la mise en place de mesures destinées à en assurer le respect. 
La Cour a jugé ce lien suffisant et analysa la question de savoir si le requérant avait été victime de discrimination dans la jouissance du droit au respect de sa vie familiale. ${ }^{71}$

En jugeant applicable l'article 8 alors que les faits en litige n'avaient qu'une incidence sur le droit qu'il consacre, la Cour a fait preuve d'une grande souplesse. Un lien plus étroit aurait cependant pu être établi avec cette dernière disposition en faisant valoir que la loi avait plus qu'une simple incidence sur le droit protégé dès lors qu'elle pénalisait le choix personnel qu'avait fait monsieur Petrovic de demeurer à la maison afin de pourvoir aux soins de son enfant, une décision qui se trouve au cœur de la vie familiale.

Il importe de remarquer ici qu'en dépit de la libéralité qu'elle démontre, la Cour européenne demeure nécessairement limitée par le libellé clair de l'article 14 qui ne consacre l'égalité, au risque de le répéter, que dans la jouissance d'un autre droit ou liberté. ${ }^{72}$

71. Petrovic c. Autriche, supra note 67 au para. 27 et s. En l'espèce, il n'y pas eu violation de ces articles puisque la différence de traitement était justifiée.

72. Certains auteurs sont toutefois d'avis que «poussée à l'extrême», la souplesse que démontre la Cour européenne dans l'application de l'article 14 est susceptible de l'ériger en norme d'égalité quasi-indépendante (J.-F. Flauss, supra note 33 aux pp. 416 et 417; P. Lambert, supra note 8 à la p. 501; M. Levinet, supra note 14 à la p. 117; F. Sudre, supra note 14 aux pp. 973 à 976; F. Sudre, «La perméabilité» de la Convention européenne des droits de l'homme aux droits sociaux», dans Pouvoir et libertés - Études offertes à Jacques Mourgeon, Bruxelles, Les Éditions Bruylant, 1998 aux pp. 474 à 478; F. Sudre, «La protection des droits sociaux par la Convention européenne des droits de l'homme», dans Les nouveaux droits de l'homme en Europe, Bruxelles, Les Éditions Bruylant, 1999, 103 à la p. 121 et s.; F. Sudre, supra note 8 à la p. 306). Ces auteurs avancent plus particulièrement que la Cour procède parfois à l'analyse d'un litige sous un angle égalitaire alors qu'aucune disposition de la Convention autre que l'article 14 n'était susceptible de s'appliquer en corrélation avec ce dernier. La Cour aurait dès lors implicitement élargit le champ d'application de l'article 14 à des droits ou libertés non garantis par la Convention. La problématique ainsi soulevée relève toutefois avec égards d'une interprétation stricte de certains droits ou libertés consacrés dans la Convention qui ne correspond pas avec le sens et la portée que la Cour européenne donne à ces dispositions qui, par ailleurs, étaient invoqués en combinaison avec l'article 14 dans les décisions sur lesquelles s'appuient les auteurs (voir : Van Raalte, supra note 8; Gaygusuz, supra note 8; Schuler-Zgraggen c. Suisse, arrêt du 24 juin 1993). La Cour n'a donc jamais ni implicitement, ni explicitement remise en question la non-indépendance de la norme européenne d'égalité. Pour une analyse détaillée des arrêts précités, voir: D. Robitaille, L'interaction entre l'article 10 de la Charte 
Les participants au $7^{\mathrm{e}}$ Colloque international sur la Convention européenne des Droits de l'Homme, tenu à Copenhague, Oslo et Lund du 30 mai au 2 juin 1990, reconnaissaient d'ailleurs les faibles possibilités de développement jurisprudentiel quant à l'application éventuelle de l'article 14 audelà des droits et libertés reconnus dans la Convention. ${ }^{73}$ Voilà probablement pourquoi le Comité des Ministres du Conseil de l'Europe adoptait en 2000 le Protocole $n^{\circ} 12$ à la Convention de sauvegarde des Droits de l'Homme et des Libertés fondamentales,$^{74}$ destiné essentiellement à pallier la limite inhérente de non-indépendance de la norme d'égalité européenne. ${ }^{75}$ L'article $1 \mathrm{du}$ Protocole $n^{\circ} 12$ prévoit en effet la création d'une norme égalitaire générale, applicable à toute distinction dans la jouissance de tout droit découlant non seulement de la Convention mais de la loi en général, notamment des différentes législations nationales européennes ${ }^{76}$ :

\section{Article 1 - Interdiction générale de la discrimination}

La jouissance de tout droit prévu par la loi doit être assurée, sans discrimination aucune, fondée notamment sur le sexe, la race, la couleur, la langue, la religion, les opinions politiques ou toutes autres opinions, l'origine nationale ou sociale, l'appartenance à une minorité nationale, la fortune, la naissance ou toute autre situation.

québécoise et les autres droits et libertés de la personne : l'influence de la Convention européenne et de la Charte canadienne, mémoire de maîtrise, Québec, Faculté des études supérieures, Université Laval, 2002 aux pp. 34 à 42.

73. Voir : CONSEIL DE L'EUROPE, Protocole $n^{\circ} 12$ à la Convention de sauvegarde des Droits de l'Homme et des Libertés fondamentales, Rapport explicatif au para. 3 (ci-après Rapport explicatif), document obtenu sur le site Internet du Conseil à l'adresse suivante : http://conventions.coe.int/Treaty/fr/Reports/Html/177.htm.

74. STE $n^{\circ}: 177$ (ci-après Protocole $n^{\circ} 12$ ).

75. Voir : CONSEIL DE L'EUROPE, Convention européenne des Droits de l'Homme : une meilleure protection contre la discrimination, document obtenu à l'adresse Internet suivante : http://press.coe.int/cp/2000/473f(2000).htm.

76. Rapport explicatif, supra note 73 au para. 22. Il importe de préciser que l'article 1 n'est pas destiné à remplacer l'article 14 mais s'ajoute à celui-ci : Rapport explicatif, supra note 73 au para. 33. Les deux garanties sont en effet complémentaires; l'article 14 continuera donc toujours de s'appliquer et, pour les États qui ne ratifieront pas le Protocole $n^{\circ} 12$, demeurera la seule norme égalitaire que pourront invoquer les justiciables. 
Nul ne peut faire l'objet d'une discrimination de la part d'une autorité publique quelle qu'elle soit fondée notamment sur les motifs mentionnés au paragraphe 1 .

(nos italiques)

L'adoption de ce Protocole constitue un effort salutaire en vue d'atteindre l'idéal que représente l'élimination de toute discrimination. Cela dit, il n'en demeure pas moins que ce document, ouvert à la signature des États membres du Conseil de l'Europe le 4 novembre 2000, n'entrera en vigueur qu'après ratification de 10 d'entre eux. $^{77}$ À l'heure actuelle, seulement 3 pays signataires sur 30 l'ont ratifié, soit la Croatie (3 février 2003), Chypre (30 avril 2002) et la Géorgie (15 juin 2001) à l'exception notable de la France et du Royaume-Uni. ${ }^{78}$ Certains États membres du Conseil de l'Europe ne semblent donc pas prêts à soumettre leurs législations nationales respectives à une norme générale d'égalité dont il est à souhaiter que la Cour européenne assurera, un jour, le respect.

\section{B. La quasi-indépendance de la norme d'égalité par l'utilisation du concept de dignité en matière de discrimination}

Contrairement à l'article 14 de la Convention, l'article 10 de la Charte a fait l'objet de développements jurisprudentiels récents qui permettent de croire à un élargissement potentiel de l'application de la norme d'égalité au-delà de sa limite inhérente de non-indépendance.

S'inspirant directement du célèbre arrêt Law c. Canada,${ }^{79}$ par lequel la Cour suprême définissait le «nouveau» cadre d'analyse de l'article 15 de la Charte canadienne, la Cour d'appel du Québec exige maintenant que le demandeur en vertu de l'article 10 de la Charte québécoise démontre que la distinction subie porte atteinte à sa dignité humaine. ${ }^{80}$ Ainsi, la preuve d'une

77. Protocole $n^{\circ} 12$, supra note 74 à l'art. 5.

78. Voir l'état des signatures et ratifications du Protocole $n^{\circ} 12$ à l'adresse Internet suivante : http://conventions.coe.int/Treaty/FR/searchsig.asp?NT=177\&CM=\&DF=.

79. [1999] 1 R.C.S. 497.

80. Amselem, supra note 35; Québec (Procureur général) c. Lambert, [2002] R.J.Q. 599 à la p. 610 (C.A.); Johnson c. Commission scolaire Lester B. Pearson/Lester B. Pearson School Board, [2000] R.J.Q. 1961 aux pp. 1970 et 1971 (C.A.) (ci-après Commission scolaire Lester B. Pearson). Les tribunaux inférieurs suivent également ce courant jurisprudentiel : 
Non-indépendance et autonomie de la norme
(2004) 35 R.D.U.S. $\quad$ d'égalité québécoise : des concepts «fondateurs»

qui méritent d'être mieux connus

distinction préjudiciable fondée sur un motif énuméré dans la reconnaissance ou l'exercice d'un droit ou d'une liberté de la personne ou d'un domaine protégé d'activités n'est désormais plus suffisante. ${ }^{81}$

Gagnon c. Office municipal d'habitation de L'Ancienne-Lorette, [2000] R.J.Q. 2728 aux pp. 2734 à 2736 (C.Q.); Ordre des comptables généraux licenciés du Québec c. Québec (Procureur général), [2001] R.J.Q. 1919 à la p. 1936 (C.S.); Champagne c. Tribunal administratif du Québec, [2001] R.J.Q. 1788 à la p. 1800 (C.S.); Gosselin c. Québec (Procureur général), [2000] R.J.Q. 2973 aux pp. 3006 et 3007 (C.S.), confirmé en appel par l'arrêt Gosselin de la Cour d'appel, supra note 16; Québec (Procureure générale) c. Entreprises W.F.H. ltée, [2000] R.J.Q. 1222 aux pp. 1229, 1250 et s. (C.S.), confirmé en appel par Québec (Procureure générale) c. Entreprises W.F.H. ltée, [2001] R.J.Q. 2557 aux pp. 2569 à 2571 (C.A.). Tel que le démontrent les propos utilisés par les magistrats dans plusieurs affaires (Corbiere c. Canada (Ministre des Affaires Indiennes et du Nord canadien), [1999] 2 R.C.S. 203 aux para. 17 et 18; Winko c. Colombie-Britannique (Forensic Psychiatric Institute), [1999] 2 R.C.S. 625 au para. 74; Commission scolaire Lester B. Pearson, 1971 et 1972), l'atteinte à la dignité consiste selon nous en réalité à déterminer si la différence de traitement dont se plaint le demandeur est raisonnable ou n'est pas arbitraire. Pour de plus amples détails à ce sujet, voir : D. Robitaille, «Vous êtes victime de discrimination et vous souhaitez en faire la preuve? Bonne chance!» (2002) 62 R. du B. 319 aux pp. 344 à 357. C'est également l'opinion des professeurs H. Brun et G. Tremblay, supra note 5 aux pp. 1113 et 1114. Le professeur Proulx, pour sa part, ne partage pas cette vue : D. Proulx, supra note 6 aux pp. 539 à 541.

81. Il est probable que les tribunaux québécois ont intégré le test de la dignité humaine à la norme d'égalité québécoise en raison de l'absence de disposition justificative expresse générale dans la Charte, c'est-à-dire applicable à toute discrimination. Dans ce contexte, il n'était pas nécessaire de procéder à un tel revirement jurisprudentiel puisque l'auteur d'une discrimination disposait déjà d'un moyen exonératoire dans la mesure où les tribunaux lui reconnaissent généralement la possibilité de démontrer que sa conduite est raisonnablement justifiable, qu'il a accommodé le plaignant ou qu'un tel accommodement lui causerait une contrainte excessive. Voir, notamment : Commission scolaire régionale de Chambly c. Bergevin, [1994] 2 R.C.S. 525 aux pp. 544 à 546 ; Amselem, supra note 35 aux pp. 915 à 922 (J. Morin); Whittom c. Commission des droits de la personne, [1997] R.J.Q. 1823 aux pp. 1825 à 1827 (C.A.) (J. Brossard); Desroches c. Commission des droits de la personne, [1997] R.J.Q. 1540 aux pp. 1554 à 1559 (C.A.); Commission-scolaire-St-Jean-surRichelieu, supra note 5 à la p. 1246; Commission des droits de la personne et des droits de la jeunesse c. Fondation Abbé Charles-Émile Gadbois, T.D.P.Q., Montréal, 500-53-000149003, 2001-08-27, J. d'Amour, AZ-50100604 aux para. 33 et 34; Commission des droits de la personne et des droits de la jeunesse c. Hôtel Villa de France, T.D.P.Q., Montréal, 50053-000073-971, 1998-02-26, J.E. 98-796 aux para. 26 à 30. Certains auteurs sont toutefois d'avis qu'en dehors des exceptions prévues, l'article 10 consacre une norme d'égalité absolue et n'est sujet à aucune norme générale de justification : C. Brunelle, Discrimination et obligation d'accommodement en milieu de travail syndiqué, Cowansville (Qc), Yvon Blais, 2001 à la p. 143; D. Proulx, supra note 3 aux pp. 461, 522 et 546 à 549; D. Proulx, supra note 12 à la p. 387; D. Proulx, supra note 6 aux pp. 539 à 541. 
Si l'ajout de cette condition d'ouverture du droit à l'égalité peut se justifier dans le contexte de la norme constitutionnelle générale d'égalité, il en va tout autrement en ce qui concerne l'article 10 de la Charte québécoise alors que cette dernière, contrairement à la Charte canadienne, consacre à son article 4 un droit indépendant à la dignité humaine ${ }^{82}:$ «Toute personne a droit à la sauvegarde de sa dignité, de son honneur et de sa réputation».

Après analyse, on s'aperçoit rapidement que la notion de dignité à laquelle fait référence cet article 4, telle que définie par la jurisprudence dont celle de la Cour suprême, rejoint pour l'essentiel la définition qu'en donne cette même Cour pour les fins de l'article 15 de la Charte canadienne dans l'arrêt $L a w^{83}$ et que la Cour d'appel a transposée à l'article 10 de la Charte québécoise. Que ce soit en vertu de l'article 15 ou de l'article 4, l'on constate en effet que la dignité humaine signifie qu'une personne ressent du respect ou de l'estime de soi et relève de l'intégrité physique et psychologique personnelle ou des attributs fondamentaux de l'être humain. ${ }^{84}$ On retrouve ainsi, dans ces deux dispositions, des notions convergentes de dignité.

82. Charte, supra note 1 à l'art. 4. Québec (Curateur public) c. Syndicat national des employés de l'hôpital St-Ferdinand, [1996] 3 R.C.S. 211 au para. 100 (ci-après Hôpital StFerdinand); Poulin, supra note 5 à la p. 9 du texte intégral ; Villa Plaisance, supra note 12 aux pp. 519 à 523 .

83. C'est d'ailleurs ce que reconnaît le Tribunal des droits de la personne : Nicolet, supra note 51 aux para. 91 et 92; Poulin, supra note 5 aux pp. 9 et 10 du texte intégral; Commission des droits de la personne et des droits de la jeunesse c. Sinatra, [2000] J.L. 45 aux pp. 51 et 52 (T.D.P.Q.); Commission des droits de la personne et des droits de la jeunesse c. Beaublanc, [1999] R.J.Q. 1875 aux pp. 1878 et 1879 (T.D.P.Q.).

84. Sur l'article 15, voir l'arrêt Law, supra note 79 au para. 53 et sur l'article 4, voir les arrêts suivants : Gauthier c. Beaumont, [1998] 2 R.C.S. 3 au para. 90 (J. Gonthier); Hôpital StFerdinand, supra note 82 au para. 105; Blanchette c. Bury, [2001] R.R.A. 904 à la p. 913 (C.S.); Marquis c. Syndicat professionnel des infirmières et infirmiers du Québec, [2001] R.R.A. 852 (rés.); Groupe Champlain inc. c. Lauzon, C.S., Montréal, 500-05060737-002, 2001-04-05, J. Rayle ; Daigneault (Succession de) c. 9045-5619 Québec inc., [2001] R.J.Q. 538 aux pp. 544 à 546 (C.Q.); Hervieux-Payette c. Société St-Jean-Baptiste de Montréal, [1998] R.J.Q. 131 à la p. 138 (C.S.), infirmé par Société St-Jean-Baptiste de Montréal c. Hervieux-Payette, [2002] R.J.Q. 1669 (C.A.), mais sur un autre point; Poulin, supra note 5 aux pp. 9 et 10 du texte intégral; Villa Plaisance, supra note 12 aux pp. 522 et 523 . 
Ce constat établi, on peut dès lors tirer la conclusion suivante : exiger une atteinte à la dignité humaine en tant que condition sine qua non pour fonder une constatation de discrimination en vertu de l'article 10 revient de manière générale à requérir une violation de l'article 4 de la Charte. Or, cette dernière disposition constitue un droit de la personne susceptible d'être invoqué en corrélation avec l'article 10. Dans cette perspective, l'exigence systématique d'une violation de la dignité en vertu de la norme d'égalité québécoise a pour effet de ramener à un automatisme l'étape consistant en l'identification d'un droit ou d'une liberté dans le cadre duquel s'opère une distinction. L'article 4 se trouvera en effet à être continuellement et nécessairement invoqué en combinaison avec l'article 10 et sert ainsi de vecteur à la quasi-indépendance de ce dernier. ${ }^{85}$

L'application de l'article 10, à l'origine un droit non-indépendant, susceptible d'interagir avec l'ensemble des droits et libertés de la personne, se trouve ainsi cantonnée aux cas de discrimination dans la reconnaissance ou l'exercice du droit à la sauvegarde de sa dignité, puisque le rattachement avec les autres droits et libertés ne sera désormais ni suffisant ni nécessaire. ${ }^{86}$

La Cour d'appel n'a certainement pas souhaité un tel résultat ni, probablement, ne l'avait envisagé lorsqu'elle décidait de suivre la Cour suprême quant à l'utilisation de la dignité en matière de discrimination. Tel est pourtant, dans l'état actuel du droit, l'imbroglio dans lequel se trouve la norme d'égalité

85. Nous employons l'expression quasi-indépendance puisqu'en dépit du jumelage systématique entre les articles 10 et 4 , il n'en demeure pas moins qu'un lien avec ce dernier droit demeurera toujours présent, la norme d'égalité ne pouvant d'ailleurs s'appliquer isolément de quelque autre droit ou liberté de la personne. Par ailleurs, si nous qualifions l'article 10 de norme indépendante lorsqu'il se rattache à un domaine d'activité, supra aux pp. 15 et 17, c'est en raison du fait qu'il n'est alors invoqué avec aucun autre droit ou liberté de la personne.

86. Dans la mesure où la dignité humaine devient l'essence de la norme d'égalité, rien n'empêcherait par ailleurs le demandeur de n'invoquer qu'une atteinte à son droit à la sauvegarde de sa dignité en vertu de l'article 4, évitant ainsi d'avoir à faire la preuve de tous les éléments nécessaires à la démonstration d'une violation du droit à la non-discrimination. Cela pourrait être avantageux lorsque la distinction dont se plaint le demandeur ne repose sur aucun motif énuméré à l'article 10. Pour éviter qu'une telle situation se produise, les tribunaux pourraient cependant conclure que le champ égalitaire de la dignité humaine dans la Charte québécoise se limite à l'article 10. 
québécoise. Nous constaterons ci-après que si cette nouvelle interprétation a pour effet de transformer l'article 10 en un droit quasi-indépendant, elle a également un effet sur l'autre facette fondamentale de cette disposition, son autonomie.

\section{L'indispensable autonomie de la norme d'égalité québécoise}

Si la norme d'égalité québécoise n'est susceptible d'aucune application indépendante, elle possède néanmoins une portée autonome. L'article 10 peut ainsi se trouver enfreint par une mesure conforme, en elle-même, aux exigences d'un autre article mais qui crée une inégalité de traitement dans la reconnaissance ou l'exercice du droit ou de la liberté consacrée par cette autre disposition. ${ }^{87}$ Il est en effet reconnu qu'une constatation de discrimination en vertu de l'article 10 ne nécessite pas une «double violation», c'est-à-dire celle du droit ou de la liberté dans le champ duquel la distinction se pratique et celle du droit à l'égalité. ${ }^{8}$

Portant sur l'article 14 de la Convention européenne, l'Affaire linguistique belge en constitue un excellent exemple et, s'agissant de l'arrêt de principe de la Cour européenne sur cette question, fait figure de précurseur en la matière. ${ }^{89} \mathrm{Se}$ fondant sur les articles 2 du Protocole $n^{\circ} 1$ (droit à l'instruction) ${ }^{90}$ et 14 de la Convention, les requérants francophones se plaignaient de la législation belge en matière d'enseignement qui les obligeait à envoyer leurs enfants étudier à l'extérieur de leur commune pour recevoir une instruction en langue française. Les enfants de parents dont la langue maternelle était néerlandaise recevaient quant à eux, dans leur commune, un enseignement dispensé dans leur langue.

87. Voir l'une des premières études sur le sujet : M.-A. Eissen, supra note 8 à la p. 123.

88. Supra note 5.

89. G. Cohen-Jonathan, supra note 14 à la p. 538; P. Lambert, supra note 8 aux pp. 500 et 501 ; F. Sudre, supra note 14 à la p. 968.

90. L'article $2 \mathrm{du}$ Protocole additionnel à la Convention de sauvegarde des Droits de l'Homme et des Libertés fondamentales, Paris, 20.III.1952 (ci-après Protocole $n^{\circ} 1$ ) énonce que : «Nul ne peut se voir refuser le droit à l'instruction. L'État, dans l'exercice des fonctions qu'il assumera dans le domaine de l'éducation et de l'enseignement, respectera le droit des parents d'assurer cette éducation et cet enseignement conformément à leurs convictions religieuses et philosophiques.» 
Dans la mesure où, selon la Cour, l'article 2 du Protocole $n^{\circ} 1$ ne garantit pas aux parents le droit pour leurs enfants de recevoir une instruction selon leurs préférences linguistiques, ${ }^{91}$ le recours fondé strictement sur cette disposition a échoué.

Le respect de l'article 2 du Protocole $n^{\circ} 1$ n'a cependant pas empêché la Cour de constater que les enfants des requérants avaient fait l'objet d'un traitement discriminatoire dans la jouissance du droit à l'instruction qu'il énonce puisque la distinction établie par la loi ne s'appliquait pas de façon uniforme aux deux groupes linguistiques en cause sur le seul fondement de la langue et du lieu de résidence des parents. ${ }^{92}$ En d'autres termes, la portée autonome de l'article 14 a permis à la Cour de sanctionner une inégalité dans la jouissance d'un droit en lui-même respecté.

Voilà qui illustre bien, par ailleurs, que les individus ne peuvent exiger la création de mesures étatiques qui satisfont leurs priorités individuelles sur la base de dispositions qui ne consacrent que l'obligation de respecter le droit ou la liberté consacré. L'État qui décide néanmoins de mettre en place des programmes qui vont au-delà des exigences limitées que prescrit le droit ne peut 

dispositions qui n'imposent à l'autorité publique qu'une obligation négative. ${ }^{94}$

La reconnaissance de cette caractéristique commune aux normes québécoise et européenne d'égalité s'avère fort heureuse puisque c'est principalement cette portée autonome qui leur donne un rôle véritable et une efficacité certaine. ${ }^{95}$ D'ailleurs, si la violation de l'article 10 nécessitait celle

93. On pense ici inévitablement à la récente décision de la Cour supérieure dans Baie d'Urfé (Ville) c. Québec (Procureur général), [2001] R.J.Q. 1589 (C.S.), que l'on peut communément appeler l'«Affaire des fusions». Certains demandeurs contestaient le fait que le législateur les avait exclus, sur la base de leur lieu de résidence, du mécanisme de consultation préalable aux fusions municipales dont bénéficiaient pourtant certains citoyens d'autres municipalités et prétendaient, par conséquent, être victimes de discrimination dans l'exercice de leur liberté d'expression. Le pourvoi a toutefois été rejeté, la Cour étant d'avis que la distinction alléguée n'était fondée sur aucun motif illicite prévu à l'article 10, résultat d'une interprétation stricte du motif énuméré d'«état civil» qui, selon cette dernière, ne comprend pas le domicile. Un parallèle intéressant peut par ailleurs être établi avec l'arrêt Haig c. Canada; Haig c. Canada (Directeur général des élections), [1993] 2 R.C.S. 995. En l'espèce, le demandeur invoquait avoir été privé de l'exercice des droits protégés aux articles 2b) (liberté d'expression) et 15 de la Charte canadienne puisqu'il avait été empêché de voter à un référendum pour des raisons administratives liées au lieu de son domicile. La Cour a conclu qu'en dépit du fait que la liberté d'expression n'oblige pas le gouvernement à mettre en place des mesures destinées à en assurer la jouissance, l'État qui décide de fournir un mode d'expression tel un référendum doit le faire de façon conforme à la Constitution dont fait partie la Charte: Haig c. Canada, 1041 (J. L'Heureux-Dubé). Voir aussi les propos de la juge L'Heureux-Dubé dans l'arrêt Association des femmes autochtones du Canada c. Canada, [1994] 3 R.C.S. 627.

94. Supra note 70.

95. Il faut toutefois reconnaitre que des violations autonomes de la norme d'égalité européenne sont rares : L.-E. Pettiti, E. Decaux et P.-H. Imbert, supra note 8 à la p. 480. Voir aussi M. Bossuyt, supra note 14 aux pp. 164 et 165. En effet, la Convention reconnait essentiellement des droits civils et politiques et des libertés fondamentales qui imposent un devoir d'abstention ou de non-ingérence à l'État, les droits économiques et sociaux relevant plutôt de la Charte sociale européenne (révisée), Strasbourg, 3.V.1996. Ce faisant, la privation discriminatoire de la jouissance d'un de ces droits ou libertés constitue souvent, mais non nécessairement, non seulement une discrimination mais également une violation du droit ou de la liberté en question. À l'opposé, dans la mesure où les motifs illicites qu'énumère l'article 14 ne sont pas exhaustifs, la privation arbitraire d'un droit ou d'une liberté implique toujours une discrimination puisque tous doivent jouir de tous les droits et libertés sans distinction : L.-E. Pettiti, E. Decaux et P.-H. Imbert, supra note 8 à la p. 480. Lorsqu'un droit ou une liberté impose une obligation positive à l'État ou lorsque l'État décide, sans y être tenu, d'aménager des moyens d'exercice d'un droit, la possibilité qu'une violation autonome de l'article 14 soit constatée augmente : Ibid. Ainsi, puisque les obligations 
concomitante d'un droit ou d'une liberté de la personne, une constatation de discrimination ne constituerait alors qu'une circonstance aggravante ${ }^{96}$ du nonrespect du droit ou de la liberté invoqué. En effet, une fois constatée l'atteinte à ce dernier, il n'est alors plus strictement nécessaire, quoique parfois opportun, ${ }^{97}$ de se demander si tous en jouissent en pleine égalité.

\section{A. L'importance capitale de la norme autonome d'égalité lorsque rattachée à un droit économique ou social}

Au Québec, l'autonomie de l'article 10 s'avère particulièrement importante en ce qui concerne certains droits économiques et sociaux, consacrés aux articles 39 à 48, lesquels font malheureusement figure de négligés dans la Charte.$^{98}$ C'est le cas du droit à l'instruction publique gratuite «dans la mesure et suivant les normes prévues par la loi», ${ }^{99}$ du droit à l'information «dans la mesure prévue par la loi», ${ }^{100}$ du droit à des mesures d'assistance financière et sociales «prévues par la loi» ${ }^{101}$ et du droit de toute personne qui travaille à des conditions de travail justes et raisonnables et qui respectent sa santé, sa sécurité et son intégrité physique «conformément à la loi». ${ }^{102}$ La présence de ces limites intrinsèques dans le texte des articles 40, 44, 45 et 46 indique ainsi qu'il revient au législateur de les mettre en œuvre. ${ }^{103}$ Pour en déterminer la portée véritable,

positives imposent généralement une obligation de moyen, l'État jouissant d'une plus grande discrétion dans l'étendue de la protection qu'il accorde, la violation propre d'un droit programmatoire est donc plus difficile à démontrer que la violation d'une disposition qui n'impose qu'une obligation d'abstention à laquelle l'autorité publique ne s'est pas conformée. La norme autonome d'égalité européenne peut dès lors jouer son rôle en permettant aux tribunaux de sanctionner la discrimination dans la reconnaissance ou la jouissance d'un droit en lui-même respecté.

96. G. Cohen-Jonathan, supra note 14 aux pp. 538 et 539; P. Lambert, supra note 8 à la p. 500 et $\mathrm{s}$.

97. Supra note 23.

98. $\quad$ Voir : P. Bosset, supra note 60 à la p. 585.

99. Charte, supra note 1 à l'art. 40.

100. Ibid. à l'art. 44.

101. Ibid. à l'art. 45 .

102. Ibid. à l'art. 46 .

103. Gosselin c. Québec (Procureur général), supra note 6 aux para. 88 à 90 et 418 à 433. 


\section{il faut donc nécessairement s'en remettre à la législation pertinente puisque c'est par l'entremise de cette dernière qu'ils prennent tout leur sens. ${ }^{104}$}

Les droits économiques et sociaux, particulièrement les articles 40, 44, 45 et 46 , se présentent donc comme des droits programmatoires ${ }^{105}$ ou comme le fruit d'un énoncé politique, ${ }^{106}$ les tribunaux n'étant pas autorisés à réviser la suffisance des mesures que le législateur choisit d'adopter. ${ }^{107}$ Par conséquent, malgré le fait qu'un individu se croyant lésé dans ses droits économiques et

104. Lévesque c. Québec (Procureur général), [1988] R.J.Q. 223 à la p. 226 (C.A.); Aide sociale - 134, [1997] C.A.S. 432 aux pp. 433 et 434; Aide sociale - 27, [1996] C.A.S. 139 et Aide sociale - 43, [1994] C.A.S. 137. En ce qui concerne la portée de l'article 40 de la Charte, voir : Commission scolaire régionale Chauveau c. Commission des droits de la personne du Québec, [1994] R.J.Q. 1196 à la p. 1204 (C.A.); Commission scolaire St-Jeansur-Richelieu, supra note 5 aux pp. 1241 et 1242; Bigonesse c. École secondaire du Mont Bruno, J.E. 96-1477 (T.D.P.Q.).

105. Gosselin c. Québec (Procureur général), supra note 6 au para. 89 (J. McLachlin); P. Bosset, supra note 60 à la p. 592.

106. P. Bosset, supra note 60 à la p. 593.

107. À cet égard, il est intéressant de prendre connaissance de la dissidence de l'Honorable Michel Robert, maintenant juge en chef du Québec, dans l'arrêt Gosselin c. Québec (Procureur général), [1999] R.J.Q. 1033 à la p. 1089 et s. (C.A.). Cette affaire concernait notamment l'interprétation de l'article 45 de la Charte québécoise auquel le juge Robert s'est montré plus ouvert à donner une certaine force contraignante. Selon lui, malgré le fait que la portée de cette disposition dépend des ressources que l'État décide d'y consacrer, elle ne saurait être restreinte à un énoncé de politique générale dont l'application se vérifie dans la loi. Il s'agirait plutôt d'un droit imposant à l'État un contenu obligatoire minimum autorisant dans certains cas un examen critique et certaines sanctions par les tribunaux. Bien sûr, le législateur conserverait une marge de manoeuvre importante dans l'établissement de programmes sociaux. Les tribunaux seraient toutefois autorisés à vérifier si la suffisance minimale nécessaire à un niveau de vie décent est respectée. Ce point de vue n'a cependant pas été retenu par la majorité de la Cour suprême saisie en appel de cette affaire dans Gosselin c. Québec (Procureur général), supra note 6. Selon les juges majoritaires, l'article 45 ne constitue qu'un droit symbolique (para. 96), qui «[...] oblige le gouvernement à établir des mesures d'aide sociale, mais soustrait au pouvoir de contrôle des tribunaux la question de savoir si ces mesures sont adéquates» au para. 88 (J. McLachlin) (nos italiques). Cela est pour le moins paradoxal et le lecteur est en droit de se demander comment sera sanctionnée l'obligation qu'impose l'article 45 à l'État et dont la Cour suprême reconnaît elle-même l'existence. La juge L'Heureux-Dubé, dissidente, souscrit pour sa part entièrement à l'analyse du juge Robert de la Cour d'appel aux para. 146 à 148. 
sociaux puisse se pourvoir devant un tribunal en vertu de l'article $49,{ }^{108}$ la portée véritable de ces derniers dans la Charte rend souvent son recours illusoire. ${ }^{109}$

Comme plusieurs d'entre eux ne possèdent en effet aucune juridicité propre et qu'il est donc difficile pour un individu d'en démontrer une atteinte en soi, l'absence d'autonomie de l'article 10 empêcherait les tribunaux de sanctionner les distinctions qui ont lieu dans les modalités des droits socioéconomiques ou qui ont une incidence sur ceux-ci.

Le meilleur exemple de ce raisonnement demeure sans doute l'arrêt Commission scolaire St-Jean-sur-Richelieu de la Cour d'appel. ${ }^{110}$

Se fondant sur les articles 10 et 40 de la Charte (droit à l'instruction publique gratuite), les demandeurs contestaient le refus des autorités scolaires de défrayer les services d'une accompagnatrice spécialisée qui aurait facilité l'intégration en classe régulière de leur enfant présentant des traits autistiques.

Après analyse du droit international et de la législation en vigueur, ${ }^{111}$ la Cour a conclu que le droit à l'intégration en classe régulière des élèves souffrant d'un handicap n'est pas un droit absolu et n'impose qu'une obligation de moyen à l'État. ${ }^{112}$ Le droit énoncé à l'article 40, directement lié aux mesures mises de l'avant par la loi, ${ }^{113}$ ne pouvait donc servir de base unique au recours des parents puisqu'en l'espèce la commission scolaire avait respecté cette obligation de

108. Bien que la majorité de la Cour suprême dans l'arrêt Gosselin c. Québec (Procureur général), supra note 6 au para. 96, ne fasse pas mention expresse de l'article 49, elle est d'avis qu' «[...] il existe une réparation pour les atteintes aux droits sociaux et économiques énoncés au chapitre IV de la Charte québécoise» et qu'en cas de «[...] violation de ces droits, un tribunal compétent peut prononcer un jugement déclaratoire constatant cette violation» (soulignement et italique dans le texte original). Les juges Bastarache (para. 304) et Arbour (para. 396) ne sont pas de cet avis ayant plutôt jugé dans cette affaire que l'article 49 ne pouvait être invoqué à l'encontre de la violation d'un droit économique ou social.

109. Certains droits énoncés aux articles 39 à 48 sont toutefois susceptibles de créer de réelles obligations. Nous référons le lecteur à l'article de P. Bosset, supra note 60 , pour de plus amples détails à ce sujet.

110. Supra note 5.

111. Loi sur l'instruction publique, L.R.Q. c. I-13.3., art. 234 et 235.

112. Commission scolaire St-Jean-sur-Richelieu, supra note 5 aux pp. 1241 et 1242.

113. Ibid. à la p. 1242 . 
moyen en mettant à la disposition des élèves présentant un handicap ou des difficultés d'apprentissage des mesures visant à faciliter leur intégration en classe régulière. L'insatisfaction des parents quant à ces mesures ne suffisait pas pour fonder une violation propre de l'article 40, comme c'était le cas d'ailleurs dans l'Affaire linguistique belge où, rappelons-nous, la Cour européenne affirmait que les ressortissants belges ne pouvaient sur la base de l'article $2 \mathrm{du}$ Protocole $n^{\circ} 1$ exiger la création d'établissements d'enseignements conformes à leurs préférences linguistiques. ${ }^{114}$

Toutefois, malgré cette conclusion quant au respect de l'article 40 par l'État, la distinction fondée sur le handicap dont avait fait l'objet l'enfant dans l'exercice de son droit à l'instruction publique gratuite était discriminatoire en ce qu'elle avait sur lui un réel effet d'exclusion. ${ }^{115}$

Il importe ici de souligner qu'en constatant la discrimination dans l'exercice d'un droit social, les tribunaux ne portent pas un regard sur la suffisance même des mesures choisies par le législateur et, dans cette perspective, n'ont pas pour mission de sanctionner la violation propre du droit dont il est question. En réalité, il s'agit plutôt d'assurer la protection d'une personne victime d'un traitement arbitraire ou déraisonnable ${ }^{116}$ différent, entretenant un certain rapport avec un droit économique ou social ${ }^{117}$ et fondé sur un motif illicite. Un auteur est toutefois d'avis que l'application de la norme d'égalité en conjonction avec le genre de droit dont il est question a un effet créateur de droit :

[...] malgré l'absence de violation du droit social, l'individu peut établir un titre juridique à l'exercice de ce droit en se fondant sur la norme de nondiscrimination. Cette dernière interdit notamment le refus pour des raisons discriminatoires de l'exercice d'un droit social reconnu dans la législation nationale et prévu par la convention. Grâce à cet effet créateur de l'application autonome de la norme de non-discrimination, l'individu peut

114. Supra note 91.

115. Commission scolaire St-Jean-sur-Richelieu, supra note 5 à la p. 1248.

116. Supra note 80.

117. Supra notes 60 et 61 . 
acquérir un droit (social) que ni la législation nationale, ni la convention internationale ne garantissent directement! ${ }^{118}$

À notre avis, l'application autonome de la norme d'égalité avec un droit social n'a aucun tel effet créateur. Bien que l'individu puisse acquérir un titre juridique à l'exercice d'un droit économique ou social qui ne lui était pas attribué au départ, il ne s'agit pas de la création d'un droit. Au contraire, ce droit est déjà prévu : celui de la non-discrimination dans la reconnaissance ou l'exercice des droits et libertés de la personne, ce qui inclut les droits économiques et sociaux. ${ }^{119}$

Certains pourraient par ailleurs prétendre que la différence de nature des droits civils et politiques et des droits économiques et sociaux devrait faire en sorte que l'application autonome de la norme d'égalité à ces derniers droits devrait être plus relative, comme le soutenait d'ailleurs le gouvernement dans l'Affaire linguistique belge. ${ }^{120}$ Nous partageons plutôt le point de vue du professeur Proulx ${ }^{121}$ selon lequel le principe d'égalité doit s'appliquer avec la même vigueur à tous les droits. En conséquence, bien que les droits socioéconomiques nécessitent une intervention de l'État et que leur mise en œuvre s'effectue progressivement dans le temps, il n'en demeure pas moins que dans

118. M. Bossuyt, supra note 14 aux pp. 218 et 219.

119. La majorité de la Cour suprême dans l'arrêt Haig c. Canada, supra note 93 à la p. 1041 affirmait d'ailleurs ceci au sujet de l'article 15 de la Charte canadienne : «[...] un gouvernement peut se voir obligé de prendre des mesures positives afin d'assurer l'égalité de personnes ou de groupes visés à l'art. 15.»

120. Supra note 8 à la p. 29 à 31. Les droits civils et politiques, considérés comme naturels et intrinsèques à la personne humaine, n'imposent généralement à l'État qu'une obligation d'abstention en ce sens qu'il doit s'abstenir d'en empêcher l'exercice : M. Bossuyt, supra note 14 à la p. 186 et s.; D. Proulx, supra note 3 à la p. 494. Il importe toutefois de préciser que ce n'est pas le cas de tous les droits civils et politiques. En effet, certains d'entre eux, comme le droit de vote ou le droit à un tribunal indépendant (Charte, supra note 1 aux art. 22 et 23), exigent, pour prendre tout leur sens, des actions positives de l'État. Les droits économiques et sociaux, pour leur part, impliquent toujours pour leur mise en œuvre une action positive (Ibid.). Cette obligation en est une de moyen, c'est-à-dire que l'État doit faire tout en son possible pour les réaliser et les rendre effectifs. C'est donc à lui que revient le choix des mesures à mettre en place.

121. D. Proulx, supra note 3 à la p. 495. Voir également : M. Caron, «Le droit à l'égalité dans le Code civil et dans la Charte québécoise des droits» (1985) 45 R. du B. 345 à la p. 366; M. Caron, supra note 3 aux pp. 45 et 46. 
leur reconnaissance ou leur exercice il lui est interdit de porter atteinte à l'égalité. Il convient d'ailleurs de noter qu'en dépit du fait que l'article 52 de la Charte exclut expressément les droits économiques et sociaux de la prépondérance dont bénéficient les droits et libertés sur les dispositions contraires d'autres lois, ${ }^{122}$ l'article 10 , lui, est visé par cette disposition. Il faut en conclure que le droit à la non-discrimination dans la reconnaissance ou l'exercice d'un droit économique ou social bénéficie d'une valeur prépondérante sur les dispositions législatives qui lui seraient contraires.

On pourrait enfin penser qu'un tel raisonnement a pour effet de contrecarrer toute action du législateur dans les programmes sociaux qu'il entend mettre en place puisque l'adoption de telles mesures conduit nécessairement à établir des distinctions. Avec égards, notre raisonnement n'entraîne selon nous aucune conséquence de ce genre. Bien qu'il soit inévitable que le législateur établisse des distinctions dans ses lois, il ne peut pour autant se permettre de discriminer, c'est-à-dire distinguer arbitrairement. ${ }^{123}$ Après tout, ne s'est-il pas lui-même imposé cette obligation en adoptant la Charte québécoise?

\section{B. L'autonomie de la norme d'égalité menacée par l'utilisation du concept de dignité en matière de discrimination et encore mal comprise par certains}

Alors qu'il s'agit d'un attribut indispensable à l'efficience même de la norme d'égalité, son autonomie semble aujourd'hui malheureusement remise en question : elle est menacée, d'une part, par l'utilisation de la dignité humaine comme condition impérative d'une discrimination et, d'autre part, elle est encore mal comprise par certains tribunaux.

D'abord, la nouvelle lecture que fait la Cour d'appel de l'article 10 est en effet susceptible de saper complètement le postulat qu'elle formulait pourtant en

122. L'article 52 de la Charte énonce : «Aucune disposition d'une loi, même postérieure à la Charte, ne peut déroger aux articles 1 à 38 , sauf dans la mesure prévue par ces articles, à moins que cette loi n'énonce expressément que cette disposition s'applique malgré la Charte.»

123. Supra note 80. 
$1994,{ }^{124}$ à savoir qu'une violation du droit à la non-discrimination ne nécessite pas celle d'une autre disposition.

Or, c'est tout le contraire qui se produit à l'heure actuelle puisque, comme nous l'avons vu, la violation même de l'article 10 nécessite désormais une violation de la dignité telle que protégée par l'article 4 de la Charte, ce qui va clairement à l'encontre de sa portée autonome. Dans la mesure où, cependant, l'effet de l'application corrélative et systématique des articles 4 et 10 a pour principale conséquence d'en faire une norme d'égalité quasi-indépendante des autres droits et libertés, le non-respect de son autonomie par l'exigence d'une violation de la dignité est un reproche qui pourrait devenir superflu. En effet, puisque l'importance de la portée autonome de l'article 10 est intimement liée à son absence d'indépendance : «Lorsque l'égalité n'est qu'une modalité de particularisation d'un autre droit, il n'est pas nécessaire que sa violation constitue une négation de ce droit pour donner ouverture à un remède». ${ }^{125} \mathrm{Si}$ cette limite de non-indépendance imposée par le législateur est repoussée, l'autonomie de l'article 10 n'a plus aucune raison d'être.

Si l'autonomie de la norme d'égalité est par ailleurs généralement bien comprise en jurisprudence, ${ }^{126}$ il n'en demeure pas moins que les décisions de quelques tribunaux inférieurs entretiennent une certaine confusion à cet égard. Dans plusieurs jugements, on semble ainsi suggérer étonnamment que la violation d'un autre droit ou d'une liberté est une condition sine qua non d'une constatation de discrimination.

Dans l'affaire Syndicat étudiant du cégep Marie-Victorin c. Collège d'enseignement général et professionnel Marie-Victorin, ${ }^{127}$ par exemple, il s'agissait de déterminer si les demandeurs, étudiants au cégep, avaient le droit de suivre des cours de «humanities» en français et, comme corollaire, si le cégep était tenu de les leur offrir. ${ }^{128}$ Ces cours n'étaient dispensés qu'en anglais, leur

124. Supra note 5.

125. Commission scolaire St-Jean-sur-Richelieu, supra note 5 à la p. 1243 (nos italiques).

126. Comme le constate d'ailleurs le professeur Proulx : D. Proulx, supra note 6 à la p. 516 .

127. Supra note 21.

128. Ibid. 
équivalent, les cours de philosophie, étant cependant offerts aux étudiants francophones dans leur langue.

À l'appui de leur demande, les étudiants invoquaient une atteinte à leur liberté d'expression et à leur droit à l'égalité fondée sur la langue ou l'origine nationale dans l'exercice de cette liberté. Sur la question de la violation du droit à l'égalité, la Cour supérieure a d'abord correctement rappelé que l'article 10 n'a pas d'application indépendante mais constitue une simple modalité de particularisation d'un autre droit. ${ }^{129}$ Ce principe établi, la Cour en tirait cependant le constat erroné suivant :

Cette précision quant à l'article 10 nous amène à partager l'opinion des défendeurs ${ }^{[130]}$ à l'effet que [sic] les demandeurs alléguant violation de leur liberté d'expression, il ne peut exister aucun manquement à leur droit à l'égalité protégé par la Charte québécoise si leur liberté d'expression n'est pas compromise. Or, on l'a constaté plus haut, elle ne l'est pas. ${ }^{131}$

(nos italiques)

Il s'agit là, malheureusement, d'une formulation douteuse qui brouille le raisonnement de la Cour. Toutefois, malgré ses apparences, cette décision ne remet pas en question l'autonomie de l'article 10 puisque c'est plutôt en raison de la non-indépendance de la norme d'égalité que celle-ci n'avait pas été violée dans les circonstances. ${ }^{132}$ La Cour avait en effet reconnu que toute activité n'est pas protégée par l'article 3 de la Charte et que le fait de participer à des cours de «humanities» en français n'était pas une activité relevant du champ de cette disposition. ${ }^{133}$

129. Ibid. à la p. 13 du texte intégral.

130. Avec respect, il est malheureux de constater que des juristes québécois plaident encore que la violation de l'article 10 nécessite celle d'un autre droit.

131. Collège Marie-Victorin, supra note 21 à la p. 13 du texte intégral.

132. Voir également l'affaire Lavigne, supra note 21. La Cour supérieure laissait entendre dans cette affaire que la violation de la liberté d'expression était nécessaire à une violation de l'article 10. Après analyse, l'on constate que l'autonomie de l'article 10 n'est pas remise en question puisque les faits ne permettaient de rattacher la norme d'égalité à aucun autre droit ou liberté de la personne ni à un domaine d'activité protégé.

133. Collège Marie-Victorin, supra note 21 à la p. 11 du texte intégral. 
À notre avis, cependant, les cours de philosophie constituent un forum ou un moyen d'expression pour les étudiants et la participation à ces cours peut constituer une modalité d'exercice du droit à la liberté d'expression. L'État n'est certes pas obligé par l'article 3 de fournir un tel forum d'expression mais s'il décide de le faire, il ne peut discriminer. En ce sens, cette décision de la Cour supérieure nous paraît se distancer du postulat formulé par la Cour d'appel dans l'arrêt Commission scolaire St-Jean-sur-Richelieu, selon lequel le lien entre la norme d'égalité et un autre droit ou liberté n'a pas à être étroit. ${ }^{134}$

Dans l'affaire Wagner c. I.N.G., Le groupe Commerce, compagnie d'assurances,${ }^{135}$ la demanderesse se plaignait de discrimination parce que sa compagnie d'assurances avait refusé de l'indemniser pour le vol dont elle avait été victime, au motif que la disparition de ses biens était louche d'autant plus qu'elle n'avait pas dévoilé, au moment de la souscription de la police, que son conjoint avait des antécédents judiciaires. Sur le fond, la Cour du Québec, analysant la possible violation de l' article 10, affirmait d'une façon erronée que l'attitude de la compagnie d'assurances «ne peut être discriminatoire face à la Charte des droits et libertés de la personne que si elle contrevient à un droit reconnu par cette Charte». ${ }^{136}$ La Cour concluait en revanche : «Or, le droit à l'assurance, ça n'existe pas en soi». ${ }^{137}$ Selon ce raisonnement, c'est vraisemblablement en raison de la non-indépendance de la norme d'égalité qu'il n'y avait pas violation de celle-ci. Le jugement de la Cour dans cette affaire nous semble toutefois incorrect et restrictif sur ce point puisque la demanderesse n'invoquait pas avoir fait l'objet de discrimination dans l'exercice du droit à l'assurance mais bien des articles 4 (sauvegarde de sa dignité) et 6 (jouissance paisible de ses biens) de la Charte. Or, d'une part, comme le rapport entre l'article 10 et une autre disposition n'a pas à être étroit, il nous semble que la Cour aurait très bien pu appliquer l'article 10 en interaction avec l'article 6, la demanderesse réclamant une indemnité pour perte de jouissance d'un bien. D'autre part, elle pouvait également l'appliquer en interaction avec l'article 12 qui interdit la discrimination dans les actes juridiques ayant pour objet des biens ou des services ordinairement offerts au public.

134. Supra note 5.

135. Supra note 21.

136. Ibid. à la p. 533 (nos italiques).

137. Ibid. 
L'arrêt Devine de la Cour suprême représente un autre exemple de la confusion créée par les tribunaux quant à la portée autonome de l'article $10 .{ }^{138}$ Rappelons-nous que dans cette affaire, la Cour avait conclu que les articles 52 et 57 de la Charte de la langue française violaient l'article 3 de la Charte québécoise mais que cette atteinte était justifiée. ${ }^{139}$ En ce qui concerne la discrimination fondée sur la langue dans l'exercice du droit à la liberté d'expression, la Cour affirmait ceci : «En l'espèce, les art. 52 et 57 créent effectivement une distinction fondée sur la langue usuelle mais n'ont pas pour effet de compromettre ou de détruire des droits garantis par l'art. 3. Ils respectent donc la Charte québécoise». ${ }^{140}$ Évidemment, cela suggérait fortement que la violation concomitante d'un autre droit ou d'une liberté était nécessaire à la constatation d'une discrimination en vertu de l'article 10 , d'où la critique virulente du professeur Daniel Proulx qu'il convient ici de citer au long :

En glissant ainsi de la destruction du droit à l'égalité à celle du droit à la liberté d'expression garanti à l'article 3, la Cour vient subtilement mais indéniablement de détourner complètement le sens des mots «ce droit» dont parle l'article 10 in fine. En somme, pour qu'il y ait discrimination dorénavant, une distinction doit non pas compromettre le droit à l'égalité, mais compromettre l'un ou l'autre des droits garantis dans la Charte. En d'autres termes, l'article 10 ne sert plus à garantir le principe d'égalité, ce qui constitue sa seule raison d'être, mais à protéger les autres droits et libertés, comme si leur proclamation dans la Charte ne suffisait pas à assurer leur reconnaissance. Ce glissement dans l'interprétation de la norme québécoise d'égalité aurat-il des conséquences sérieuses? Qu'on en juge. Si le deuxième alinéa de l'article 10 signifie qu'une distinction ne peut être jugée discriminatoire qu'à la condition de porter en même temps atteinte à un autre droit reconnu dans la Charte québécoise, comme l'exige la Cour suprême, on est ni plus ni moins forcé de conclure que l'article $10 \mathrm{n}$ 'a plus aucun sens en droit public $[\ldots]^{141}$

138. Devine, supra note 35.

139. Supra note 39.

140. Devine, supra note 35 à la p. 819 (nos italiques).

141. D. Proulx, supra note 12 aux pp. 392 et 393. 
Si le professeur Proulx avait raison de nous mettre en garde contre l'interprétation de l'article 10 que semblait suggérer la Cour suprême, nous ne sommes pas convaincus que la critique qu'il adressait au plus haut tribunal du pays ne résulte d'une lecture adéquate de l'arrêt Devine. Le passage du jugement contre lequel il s'insurgeait doit en effet être replacé dans son contexte. Dans l'arrêt en question, la violation de l'article 3 était justifiée. Or, cela avait nécessairement pour conséquence de contrecarrer l'application de l'article 10 puisqu'elle dépend intrinsèquement de celle des autres clauses normatives de la Charte. Le passage de l'arrêt Devine cité ci-haut ne peut alors se lire sans celuici qui vient juste avant dans le jugement :

Lorsque, comme en l'espèce, l'art. 9.1 a pour effet de limiter la portée de la liberté d'expression que garantit l'art. 3, l'art. 10 ne peut être invoqué pour contourner les limites raisonnables à cette liberté et y substituer une garantie absolue de liberté d'expression. ${ }^{142}$

En effet, lorsque la violation du droit à l'égard duquel se pratique la discrimination est justifiée, le citoyen ne peut plus prétendre à la reconnaissance et à l'exercice de ce droit, ni même ne peut-il alléguer subir une inégalité dans les modalités ou le cadre de ce dernier. Comme l'article 10 ne s'applique qu'à l'égard de la reconnaissance et l'exercice d'un droit, il ne peut alors plus s'appliquer. En d'autres termes, si le législateur est justifié de ne pas accorder un droit ou une liberté dans un cas particulier et que, pour cette raison, la personne qui se plaint ne peut légitimement revendiquer le droit ou la liberté en question, l'article 10 n'a plus de possibilité d'application puisqu'il est, dans son essence, dépendant des autres dispositions de la Charte. La question de son autonomie ne se pose alors plus puisqu'elle suppose l'appui simultané de la norme d'égalité à une autre disposition. C'est dans ce contexte, estimons-nous, qu'il faut comprendre le raisonnement de la Cour suprême dans l'arrêt Devine. Les termes employés pour l'expliquer, il faut le reconnaître, prêtaient à confusion. 
Enfin, il importe d'admettre que la Cour d'appel ${ }^{143}$ et le forum spécialisé sur la Charte qu'est le Tribunal des droits de la personne ont bien compris comment se déploie l'autonomie de l'article $10 .{ }^{144}$ Seules la Cour supérieure et la Cour du Québec maintiennent aujourd'hui le juriste dans une certaine confusion en utilisant des termes et un raisonnement qui laissent perplexe, confusion que seule une analyse vigilante permettait d'éclaircir.

\section{CONCLUSION}

L'article 10 de la Charte québécoise, comme l'article 14 de la Convention européenne, consacre le droit à la reconnaissance et à l'exercice, en pleine égalité, des droits et libertés de la personne. Le droit à la nondiscrimination entretient ainsi une relation particulière avec les autres droits et libertés garantis. En raison de cette association nécessaire avec les autres dispositions de la Charte, la norme d'égalité n'est donc pas susceptible d'application indépendante, trait distinctif fondamental qui engendre certaines conséquences pratiques qui ont été mises en exergue.

Dans le but probable de pallier cette limitation inhérente de la norme d'égalité, les tribunaux interprètent par ailleurs avec libéralité le lien devant unir les faits en litige à un autre droit ou liberté susceptible de se rattacher à l'article 10. C'est également le cas en ce qui concerne l'article 14 de la Convention, d'où l'utilisation de la jurisprudence de la Cour européenne dans la démonstration de l'application large et libérale du principe de non-indépendance de la norme égalitaire québécoise.

Si les articles 10 et 14 ne sont susceptibles d'aucune application en dehors du cadre restreint des droits et libertés reconnus par la Charte ou la Convention, la constatation d'une discrimination ne nécessite pas la violation d'une autre disposition, d'où leur portée autonome. Cette deuxième facette d'application de la norme d'égalité est indispensable à son efficacité et à sa raison d'être, particulièrement lorsque, dans la Charte, elle est invoquée en

143. Sauf la dissidence de l'Honorable Deschamps, maintenant à la Cour suprême, dans l'arrêt Habachi, supra note 5 à la p. 2538, qui semble laisser entendre que la violation de l'article 10 nécessite celle d'un autre droit ou liberté.

144. Supra note 126. 
Non-indépendance et autonomie de la norme
(2004) 35 R.D.U.S. d'égalité québécoise : des concepts «fondateurs»
qui méritent d'être mieux connus

corrélation avec certains des droits économiques ou sociaux dont une violation propre est pratiquement impossible à démontrer.

L'article 10 de la Charte a récemment été l'objet d'une «modification jurisprudentielle», la Cour d'appel exigeant désormais une atteinte à la dignité en tant que condition normative pour en arriver à un constat de discrimination. Dans l'état actuel du droit, cette nouvelle interprétation a pour effet de saboter le libellé pourtant clair de l'article 10 dans la mesure où, à l'origine une norme non-indépendante, il devient un droit quasi-indépendant par son rattachement systématique et nécessaire à l'article 4 de la Charte qui consacre le droit à la sauvegarde de sa dignité. Paradoxalement, la nouvelle lecture que fait la Cour d'appel de l'article 10 a également pour effet de menacer sa portée autonome puisque, normalement, la discrimination ne nécessite pas la violation concomitante d'un autre droit ou liberté.

Enfin, et en dépit du principe qui précède, quelques tribunaux affirment encore qu'une discrimination au sens de l'article 10 ne va pas sans la compromission ou la destruction du droit ou de la liberté dans les modalités duquel la distinction contestée se pratique. Sans pour autant remettre véritablement en question l'autonomie de la norme, certaines décisions utilisent ainsi malgré tout un langage inadéquat susceptible de confondre le juriste, confusion qu'une lecture attentive a permis d'élucider.

Le présent texte a ainsi voulu expliciter deux aspects «fondateurs» de l'article 10 dont l'étude s'avère absolument nécessaire à la compréhension de son rôle véritable dans la Charte québécoise au regard des autres dispositions qu'elle consacre. Une fois énoncés les développements qui précèdent, une question nous vient inévitablement à l'esprit : ne serait-il pas plus simple et efficace d'adopter une norme générale d'égalité? 